\title{
O Prêmio Nobel de Física de $2015^{+*}$
}

\author{
José Maria Filardo Bassalo ${ }^{1}$ \\ Academia Paraense de Ciências \\ Belém - PA
}

\section{Resumo}

Neste artigo, trataremos do Prêmio Nobel de Física de 2015, concedido aos físicos: o japonês Takaaki Kajita (n.1959) e o canadense Arthur Bruce McDonald (n.1943) pela descoberta da oscilação dos neutrinos, a qual mostra que os neutrinos possuem massa.

Palavras-chave: Prêmio Nobel de Física de 2015; Kajita e McDonald; Oscilação de neutrinos; Massa dos neutrinos.

\begin{abstract}
In this article, we will address the 2015 Nobel Prize in Physics awarded to the physicists: the japanese Takaaki Kajita (n.1959) and the canadian Arthur Bruce McDonald (n. 1943) for the discovery of neutrino oscillations, which shows that neutrinos have mass.
\end{abstract}

Keywords: 2015 Physics Nobel Prize; Kajita and McDonald; Neutrino oscillations; Neutrinos' mass.

O Prêmio Nobel de Física de 2015 (PNF/2015) foi concedido aos físicos: o japonês Takaaki Kajita (n.1959) e o canadense Arthur (“Art”) Bruce McDonald (n.1943) pela descoberta da oscilação dos neutrinos, por intermédio de experiências envolvendo os neutrinos atmosféricos (produzidos por raios cósmicos) (Kajita) e os neutrinos solares (McDonald), experiências essas que indicam serem os neutrinos possuidores de massa. Também registraremos

\footnotetext{
+ The 2015 Physics Nobel Prize

* Recebido: março de 2016. Aceito: abril de 2016.

${ }^{1}$ E-mail: jmfbassalo@gmail.com
} 
outras experiências com neutrinos de aceleradores (ou reatores) nucleares (inclusive com a participação de físicos brasileiros), com a mesma indicação.

Iniciemos este artigo com um breve registro sobre a vida deles. Kajita nasceu em 09 de março de 1959, em Higashimatsuyama, Saitama (Japão), tendo concluído seu Ensino Médio (High School), no Saitama Prefectural Kawagoe e bacharelou-se em Física pela Saitama Universidade, em 1981. Depois, foi para a Universidade de Tókio (UT), para realizar seu Mestrado em Física e, na mesma, defendeu sua Tese de Doutorado, em 1986, sob a orientação do físico japonês Masatoshi Koshiba (n.1926; PNF, 2002), então Diretor do Institute for Cosmic Ray Research (ICRR/UT). Em 1988, Kajita passou a pertencer a esse Instituto, no qual se tornou Professor Assistente, em 1992, e Professor Titular, em 1999, o que lhe permitiu dirigir o Center for Cosmic Neutrinos (CCN/ICRR/UT). É interessante destacar que Kajita recebeu os seguintes prêmios ("prizes"): 1) Asahi Prize, da Asahi Shimbun Foundation, Japão, em 1988; 2) Bruno Rossi Prize, da American Astronomical Society/High Energy Astrophysics, Estados Unidos da América, em 1989; 3) Nishina Memorial Prize, da Nishina Memorial Foundation, Japão, em 1999; 4) W. K. H. Panofsky Prize in Experimental Particle Physics, da American Physical Society/Stanford University, em 2002; 5) Japan Academy Prize, em 2012; 6) Nobel Prize in Physics, em 2015: Discovery of Atmospheric Neutrino Oscillations (Kajita Nobel Lecture, 08 de dezembro de 2015); e 7) Fundamental Physics Prize, pela Fundamental Physics Prize Foundation [fundada pelo físico e empresário russo Yuri Borisovich (Bentsionovich) Milner (n.1961), em julho de 2012], em 2016².

McDonald nasceu em 29 de agosto de 1943, em Sydney, Nova Scotia (Canadá). Na Dalhousie University desta sua cidade natal, ele obteve o título de Bacharel em Física, em 1964, e o de Mestre em Física, em 1965. Seu título de Doutor em Física foi-lhe atribuído pelo California Institute of Technology (CALTECH), em 1969, após defender sua Tese intitulada Excitation Energies and Decay Properties of $\mathrm{T}=3 / 2$ States in ${ }_{8} \mathrm{O}^{17},{ }_{9} \mathrm{~F}^{17}$ and ${ }_{11} \mathrm{Na}^{21}$, sob a orientação do astrofísico norte-americano William Alfred Fowler (1911-1995; PNF, 1983). Entre 1970 e 1982, McDonald trabalhou no Chalk River Nuclear Laboratories, em Ottawa, no Canadá e, entre 1982 e 1989, foi Professor de Física da Princeton University. Em 1989, ele foi para a Queen's University em Kingston (Ontário, Canadá), na qual rege a Gordon and Patricia Gray Chair Particle Astrophysics e, desde 1990, dirige o Sudbury Neutrino Observatory (SNO), que é um detector de água pesada a uma profundidade de $2.100 \mathrm{~m}$, localizado na Inco Nickel Mine, em Sudbury, também em Ontário, como falamos acima ${ }^{3}$. Note-se que McDonald recebeu destacadas honrarias, tais como: 1) T. W. Bonner Prize in Nuclear Physics, da American Physical Society, em 2001; 2) Bruno Pontecorvo Prize in Particle Physics, Dubna, Rússia, em 2004; 3) Officer of the Order of Canada, em 2006; 4) Benjamin Franklin Medal, do Franklin Institute, Philadelphia, nos Estados Unidos da América, em 2007; 5) Fellow of the Royal Society, Inglaterra, em 2009; 6) Killam Prize in the Natural Sciences, do Canada Council, em 2009; 7) Henry

\footnotetext{
2 <en.wikipedia.org/Kajita>.

3 <en.wikipedia.org/Kajita/McDonald>.
} 
Marshall Tory Medal, da Royal Society of Canada, em 2011; 8) Order of Ontario, em 2012; 9) Nobel Prize in Physics, em 2015:The Sudbury Neutrino Observatory: Observation of Flavor Change for Solar Neutrinos (McDonald Nobel Lecture, 08 de dezembro de 2015); e 10) Fundamental Physics Prize, pela Fundamental Physics Prize Foundation, em $2016^{4}$.

Em seguida, para entendermos a razão da concessão do PNF/2015, faremos um pequeno histórico sobre os neutrinos e suas oscilações e, para isso, usaremos como base o artigo: Neutrino Oscillations (Scientific Background on the Nobel Prize in Physics 2015, The Royal Swedish Academy of Sciences). Inicialmente, vejamos como surgiu a ideia da partícula neutrino. Em 18985, o físico neozelandês-inglês Sir Ernest Rutherford (1871-1937; PNQ, 1908) mostrou que os "raios Becquerel" [descobertos pelo físico francês Antoine Henri Becquerel (1852-1908; PNF, 1903)], em $1896^{6}$ eram constituídos de partículas carregadas positivamente [partículas (raios) alfa $(\alpha)$ ], identificada por Rutherford e pelo químico inglês Thomas Royds (1884-1955), em 19097, como o átomo de hélio (He)] e negativamente [partículas (raios) beta $(\beta)$ ]. Logo depois, em $1899^{8}$, Becquerel e, posteriormente, em $1900^{9}$, o casal Curie [o físico francês Pierre (1859-1906; PNF, 1903) e a física e química polonesa-francesa Marie Sklodowska (1867-1934; PNF, 1903; PNQ, 1911)] mostraram que as partículas $\beta$ eram elétrons (e $\mathrm{e}^{-}$) emitidos por um núcleo A que se transformava em um outro núcleo B. É oportuno registrar que, ainda em 1900 o físico francês Paul Villard (1860-1934) observou a existência de uma "terceira partícula" nãocarregada, altamente penetrante e denominada por Rutherford de raios gama $(\gamma)$. Por sua vez, ao estudar o decaimento $\beta$ (beta decay), o físico inglês Sir James Chadwick (1891-1974; PNF, 1935) estabeleceu, em $1914^{11}$, que as partículas $\beta$ possuíam um espectro contínuo de energia. Em vista disso, na década de 1920, desenvolveu-se uma questão polêmica relacionada à energia dessas $\beta$. Desejava-se saber se essa energia era determinada pelas energias dos núcleos "mãe" e "filho" ou se variava continuamente. Além do mais, havia uma questão objetiva: se um elétron $\left(\mathrm{e}^{-}\right)$é emitido por A, que se transforma em B e esse elétron, tem energia menor do que as energias de repouso desses dois núcleos, para onde vai a energia que está faltando? Essa polêmica foi resolvida pelo físico austro-norte-americano Wolfgang (Ernst) Pauli Junior (1900-1958; PNF, 1945) ao escrever, em 04 de dezembro de 1930, uma carta aos físicos, a sueco-austríaca

\footnotetext{
4 <en.wikipedia.org/McDonald/Curriculum_Vitae>.

5 Proceedings of the Cambridge Philosophical Society, v. 9, p. 401, 1898.

${ }^{6}$ Comptes Rendus Hebdomadaires des Séances de l’Académie des Sciences de Paris (CRHSASP), v. 122, p. $420 ; 501,1896$.

${ }^{7}$ Philosophical Magazine, v. 17, p. 281, 1909.

8 CRHSASP, v. 129, p. 996; 1205; 1899.

${ }^{9}$ CRHSASP, v. 130, p. 647, 1900.

10 CRHSASP, v. 130, p. 1010; 1178, 1900.

11 Verhandlungen der Deustschen Physikalische Gesellschaft, v. 16, p. 383, 1914.
} 
Lise Meitner (1878-1968) e o alemão Hans (Johannes) Wilhelm Geiger (1882-1945), que participavam da reunião do Group of Radioactivity of Tübingen. Nessa carta (intitulada: To Radioactivity Ladies and Gentlemen), ele propunha a existência de uma partícula neutra, de massa muito pequena, não excedendo um centésimo da massa do próton, emitida junto com o elétron no decaimento $\beta$. Note-se que essa proposta foi apresentada por Pauli na Reunião da Sociedade Americana de Física, realizada em junho de 1931, em Pasadena, e publicada ainda em $1931^{12}$. Essa partícula pauliana foi denominada de neutrino (v) ("nêutron pequenino", em italiano) pelo físico ítalo-norte-americano Enrico Fermi (1901-1954; PNF, 1938), em 193413, por ocasião em que formulou a teoria matemática do decaimento $\beta$, segundo a qual, por intermédio de uma nova força na natureza - chamada mais tarde de força fraca - o nêutron (n) transforma-se em um próton (p), com a emissão de um elétron ( $\mathrm{e}^{-}$) e da partícula pauliana, ou seja: $\mathrm{n} \rightarrow \mathrm{p}+\mathrm{e}^{-}+$ v. Registre-se que, ainda em 1934 (e em trabalhos independentes), o físico italiano Gian Carlo Wick (1909-1992) ${ }^{14}$ e os físicos, o germano-norte-americano Hans Albrecht Bethe (1906-2005; PNF, 1967) e o inglês Rudolf Ernest Peierls (1907-1995) ${ }^{15}$ propuseram o decaimento $(\beta)$ inverso, traduzido pela reação nuclear: $\mathrm{p} \rightarrow \mathrm{n}+\mathrm{e}^{+}+v$, onde $\mathrm{e}^{+}$é o pósitron que fora previsto pelo físico inglês Paul Adrien Maurice Dirac (1902-1984; PNF, 1933), em trabalhos publicados em $1928^{16}$, como sendo a antipartícula do elétron e que foi detectada, em $1932^{17}$, pelo físico norte-americano Carl David Anderson (1905-1991; PNF, 1936). Note-se que, em sua Nobel Lecture (12 de dezembro de 1936) (The Production and Properties of Positrons), Anderson denominou-a de pósitron.

É interessante registrar que, ainda em 193218, Chadwick descobriu o nêutron (n) como uma partícula constituinte do núcleo atômico rutherfordiano, e que, também em 193219, os químicos norte-americanos Harold Clayton Urey (1893-1981; PNQ, 1934), Ferdinand Graft Brickwedde (1903-1989) e George Moseley Murphy (1903-1969), encontraram um isótopo do hidrogênio $\left({ }_{1} \mathrm{H}^{2}\right)$, denominado por eles de dêuteron $\left({ }_{1} \mathrm{D}^{2}\right)$. Merece ainda destaque o fato de que, em 193520, o físico japonês Hideki Yukawa (1907-1981; PNF, 1949) sugeriu que as partículas do núcleo atômico (próton e nêutron) eram mantidas juntas por intermédio de uma força nuclear (denominada mais tarde de força forte) ${ }^{21}$.

\footnotetext{
12 Physical Review, v. 38, p. 579, 1931.

13 Nuovo Cimento, v. 11, p. 1, 1934; Zeitschrift für Physik, v. 88, p. 161, 1934.

14 Atti Rendiconti Lincei. Accademia Nationale dei Lincei, v. 19, p. 319, 1934.

15 Nature, v. 133, p. 532, 1934.

16 Proceedings of the Royal Society of London, A117; A118, p. 610; 351, 1928.

${ }^{17}$ Proceedings of the Royal Society of London, A41, p. 405; Science, v. 76, p. 238, 1932.

18 Nature, v. 129, p. 312, 1932.

${ }^{19}$ Physical Review, v. 39, p. 164; 864; 40, p. 1, 1932.

${ }^{20}$ Proceedings of the Physical Mathematics Society of Japan, v. 17, p. 48, 1935.

21 BASSALO, J. M.; CARUSO, F. Dirac/Fermi/Pauli. São Paulo: Livraria da Física, 2013.
} 
Proposto o neutrino (v), restava mostrar a sua existência. Uma das primeiras ideias de sua realidade no Universo aconteceu com o modelo dos neutrinos solares, proposto por Bethe e pelo físico norte-americano Charles Louis Critchfield (1910-1994) ao apresentarem, em $1938^{22}$, o famoso ciclo próton-próton $\left({ }_{1} \mathrm{H}^{1}{ }_{-1} \mathrm{H}^{1}\right)(\mathrm{CPP})$ como gerador de energia das estrelas tão (ou menos) massivas quanto o Sol, obedecendo a uma reação nuclear em que dois prótons $\left({ }_{1} \mathrm{H}^{1}\right)$ colidem formando o ${ }_{1} \mathrm{D}^{2}$, com a emissão do $\mathrm{e}^{+} \mathrm{e}$ do $v$ [devido ao decaimento $(\beta)$ inverso $\mathrm{e}$ denominado então de neutrino estelar]. Em seguida, o ${ }_{1} \mathrm{D}^{2}$ colide com $\mathrm{um}_{1} \mathrm{H}^{1}$ formando um isótopo do hélio $\left({ }_{2} \mathrm{He}^{3}\right)$ e emitindo $\gamma$. Por fim, dois ${ }_{2} \mathrm{He}^{3}$ colidem, formando ${ }_{2}{ }_{2} \mathrm{He}^{4}$, reproduzindo os dois ${ }_{1} \mathrm{H}^{1}$ (daí o CPP) e mais $24,7 \mathrm{MeV}\left[1 \mathrm{MeV}=10^{6} \mathrm{eV}\right.$, sendo $1 \mathrm{eV}$ a energia eletrostática de um elétron (e) sob a diferença de potencial de 1 volt (V)] de energia. É interessante frisar que, ainda em 1938²3, o físico alemão Barão C(K)arl Friedrich von Weizsäcker (1912-2007) propôs o outro famoso ciclo carbono-nitrogênio-oxigênio (CCNO) como gerador de energia $(24,7 \mathrm{Mev})$ das estrelas mais massivas do que o Sol, ciclo esse confirmado por Bethe, em 193924. Outra evidência do neutrino solar foi apresentada, em 194125, pelos físicos, o russonorte-americano George Antonovich Gamow (1904-1968) e o brasileiro Mário Schenberg (1914-1990) ao publicarem um famoso trabalho para explicar o mecanismo do colapso estelar. Segundo eles, quando o centro de uma estrela atinge uma densidade muito alta, começa a haver a captura de elétrons $\left(\mathrm{e}^{-}\right)$por parte dos prótons (p), que se transformam em nêutrons (n) e emitem neutrinos $(v)$, numa reação nuclear decorrente do decaimento $(\beta)$ inverso e caracterizada por: $\mathrm{p}+\mathrm{e}^{-} \rightarrow \mathrm{n}+v$. Portanto, esse mecanismo [denominado por eles de Ultra Rapid CAtastrophe (processo URCA)] é o que causa a fuga de neutrinos estelares que provoca o resfriamento estelar e, consequentemente, o seu colapso.

Desse modo, a detecção do hipotético neutrino poderia ocorrer em experiências envolvendo a sua captura por nêutrons $\left(v+n \rightarrow p+e^{-}\right)$conforme foi sugerido pelo físico ítalo-inglêsrusso Bruno Maximovitch Pontecorvo (1913-1993), em 1946 (Chalk River Laboratory Report PD-205). Assim, segundo Pontecorvo, a captação de $v$ [solares ou de reações de fissão nuclear ocorrida em reatores como, por exemplo, o Brookhaven National Laboratory (BNL), localizado em Upton, New York, criado em 1947] pelo cloro $\left({ }_{17} \mathrm{Cl}^{37}\right)$ produziria o argônio $\left({ }_{18} \mathrm{Ar}^{37}\right)$, com a emissão da $\beta$ (e $\mathrm{e}^{-}$, acompanhado de um recuo do $C \ell$. Logo depois, em 194726, Pontecorvo propôs um novo tipo de captura de neutrinos por nêutrons, com a produção de "elétrons pesados" [nova partícula fortemente ionizante e com massa em torno de 200 vezes a massa do elétron $\left(\mathrm{m}_{\mathrm{e}}\right)$, descobertos, em 1937, em experiências independentes realizadas pelos físicos, os norte-

\footnotetext{
22 Physical Review, v. 54, p. 248, 1938.

23 Physikalische Zeitschrift, v. 39, p. 633, 1938.

${ }^{24}$ Physical Review, v. 55, p. 434, 1939.

25 Physical Review, v. 59, p. 539, 1941.

26 Physical Review, v. 72, p. 246, 1947.
} 
americanos Anderson e Seth Henry Neddermeyer (1907-1988)27; e Jabez Curry Street (19061989) e Edward Carl Stevenson (n.1907) ${ }^{28}$ e denominados, posteriormente, de múons $\left.\left(\mu^{-}\right)\right]$, em reação do tipo: $v+\mathrm{n} \rightarrow \mathrm{p}+\mu^{-} \rightarrow \mathrm{p}+\mathrm{e}^{-}+\gamma$. Contudo, em 194829, o físico canadense E. P. ("Ted") Hincks e Pontecorvo não confirmaram o decaimento proposto por Pontecorvo $\left(\mu^{-} \rightarrow \mathrm{e}^{-}\right.$ $+\gamma$ ). Note-se que, também em 1948 ${ }^{30}$, o físico norte-americano Horace Richard Crane (19072007) apresentou uma revisão sobre o neutrino e sua possível detecção. É oportuno destacar que, ainda em 1948 (Centennial Meeting of the American Association for Advancement of Science) e, em 194931, os físicos, o norte-americano John Archibald Wheeler (1911-2008) e o brasileiro Jayme Tiomno (1920-2011) demonstraram que o decaimento do $\mu^{-}$envolve dois neutrinos: $\mu^{-} \rightarrow \mathrm{e}^{-}+v_{1}+v_{2}$. Note-se que, também em $1948^{32}$, o físico italiano Giampietro Puppi (1917-2006) havia previsto esse decaimento para o $\mu^{-}$, daí esse mecanismo haver sido conhecido como o Triângulo de Puppi-Wheeler-Tiomno (TP-W-T) [John Archibald Wheeler e Kenneth William Ford, Geons, Black Holes \& Quantum Foam: A Life in Physics (W. W. Norton, 1998)]. É oportuno registrar que o múon ( $\left.\mu^{-}\right)$é resultante do decaimento do píon $\left(\pi^{-}\right)$, como inicialmente foi observado, em 1947 (Nature 159, p. 694), na famosa experiência realizada pelos físicos, os ingleses Sir Cecil Frank Powell (1903-1969; PNF, 1950) e Hugh Muirhead (1925-2007), o italiano Guiseppe Pablo Stanislao Occhialini (1907-1993) e o brasileiro César (Cesare) Mansuetto Giulio Lattes (1925-2005), na qual mostraram que os raios cósmicos [basicamente, prótons (p)] ao colidirem com a atmosfera (contendo também p), produziam dois tipos de mésons carregados: "primário" [hoje: píon $\left(\pi^{+,-, 0}\right)$ ] e "secundário" [hoje: múon $\left(\mu^{+,-}\right)$], sendo este decorrência do decaimento do "primário", em uma reação nuclear e hoje caracterizada como sendo do tipo: $\mathrm{p}+\mathrm{p}=\mathrm{p}+\mathrm{n}+\pi^{+}$, com: $\pi^{+} \rightarrow \mu^{+}+v_{\mu}$.

A produção de novas partículas, decorrentes da colisão de raios cósmicos com a atmosfera foi também observada, em 194733, pelos físicos ingleses George Dixon Rochester (1908-2001) e Clifford Charles Butler (1922-1999), ocasião em que encontraram trajetórias em forma de $\mathrm{V}$ oriundas de uma origem comum, daí as denominaram de partículas $V$. Tais partículas que apresentavam um comportamento "estranho", pois elas são produzidas por força (interação) forte (vida média $\sim 10^{-23} \mathrm{~s}$ ), entre píons $(\pi)$ e nucleóns (p e $\mathrm{n}$ ) [este nome foi cunhado pelo físico dinamarquês Christian Möller (1904-1980), em $1941^{34}$ e decaíam por força (interação) fraca (vida média $\sim 10^{-10} \mathrm{~s}$ ). Nas décadas de 1950 e 1960, novas partículas "estranhas"

\footnotetext{
27 Physical Review, v. 51, p. 884, 1937.

28 Physical Review, v. 51, p. 1005, 1937.

${ }^{29}$ Physical Review, v. 73, p. 257; 1122, 1948.

${ }^{30}$ Reviews of Modern Physics, v. 28, p. 278, 1948.

${ }^{31}$ Reviews of Modern Physics, v. 21, p. 144; 153, 1949.

32 Nuovo Cimento, v. 5, p. 587, 1948.

33 Nature, v. 160, p. 855, 1947.

${ }^{34}$ Köngelise Danske Videnskab Selskab Matematisk-Fysiske Meddelanden, v. 18, p. 3, 1941.
} 
[algumas delas depois conhecidas como mésons káons $\left(\mathrm{K}^{+,-, 0}\right)$ ] foram produzidas em laboratórios mundiais: Stanford Linear Accelerator Center (SLAC) (Estados Unidos); Conseil Européen pour la Recherche Nucléaire (CERN) (Suíça/França); Fermi National Accelerator Laboratory (FERMILAB) (Estados Unidos); Frascati National Laboratory (FrNL) (Itália); BNL etc.

Voltemos aos neutrinos. Na primavera de 1948, o físico norte-americano Raymond Davis Junior (1914-2006; PNF, 2002) foi trabalhar no Departamento de Química do BNL e, ao se apresentar ao seu Diretor, o químico norte-americano Richard W. Dodson (1915-2002), perguntou-lhe o que esperava de seu apresentador. Para sua surpresa, Dodson mandou que Davis Junior fosse à Biblioteca e procurasse um tema de pesquisa. Assim, encontrou o artigo de Crane e, ao lê-lo, viu a possibilidade de "detectar" neutrinos por intermédio da reação envolvendo cloro e argônio (C $\ell$-Ar). Assim, em 1951, Davis Junior passou a usar os $v$ do reator Brookhaven Graphite Research Reactor (BGRR), do BNL enviando-os para um tanque de 3.800 litros $(\ell)$ contendo tetraclorido de carbono $\left(\mathrm{CC}_{4}\right)$. Contudo, não houve produção de argônio como era esperado, pois os reatores emitem antineutrinos e não neutrinos, como será investigado mais adiante. Em 195235, Davis Junior usou a proposta de Pontecorvo sobre o recuo de um núcleo ao receber o $v$, porém, trabalhou com a reação lítio-berílio: ${ }_{3} \mathrm{Li}^{7}+v \leftrightarrow{ }_{4} \mathrm{Be}^{7}+\mathrm{e}^{-}$(lembrar que: $v+\mathrm{n} \leftrightarrow \mathrm{p}+\mathrm{e}^{-}$), na qual um feixe de $v$ com a energia de $0,862 \mathrm{MeV}$ provoca um recuo do Li com a energia característica de $57 \mathrm{eV}$. Note-se que essa reação também pode ser interpretada como a captura de elétrons pelo Be, produzindo o Li e um feixe de $v$ com a energia de 0,862 $\mathrm{MeV}$, conforme Davis Junior considerou. É interessante destacar que P. B. Smith e J. S. Allen já haviam realizado uma experiência desse tipo, antes, em 195136. É oportuno registrar que o uso do $\mathrm{CC}_{4}$ como detector de neutrinos foi proposto, pela primeira vez, em 1945, pelo físicoquímico francês Jules Guéron (1907-1990) [Frank Close, HALF-LIFE: The Dividided Life of Bruno Pontecorvo, Physicist or Spy (Basic Books, 2015)].

Em 195337, os físicos norte-americanos Emil John Konopinski (1911-1990) e Hormoz Massou Mahmoud (n.1918) mostraram que a partícula pauliana era uma antipartícula, o hoje antineutrino do elétron $\left(\bar{v}_{\mathrm{e}}\right)$. Observe-se que esse trabalho permitiu reconhecer os neutrinos propostos por Wheeler e Tiomno ( $v_{1}$ e $\left.v_{2}\right)$ como sendo: $\bar{v}_{e}$ e $v_{\mu}$. É interessante registrar que, desde 1945, o físico norte-americano Frederick Reines (1918-1998; PNF, 1995) tentava observar a partícula pauliana sem, contudo, lograr êxito. Em 1951, ao entrar de licença sabática de seu emprego no Laboratório de Los Alamos, Reines convidou seu colega, o também físico norte-americano Clyde Lorrain Cowan Junior (1919-1974), para irem à busca daquela partícula

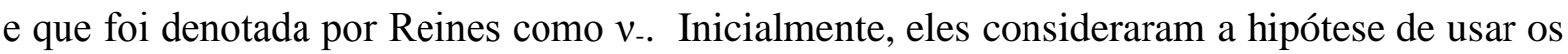
testes de bombas atômicas como fonte de neutrinos, mas logo decidiram estudar a colisão de

\footnotetext{
35 Physical Review, v. 86, p. 976, 1952.

36 Physical Review, v. 81, p. 381, 1951.

${ }^{37}$ Physical Review, v. 92, p. 1045, 1953.
} 
um fluxo de "neutrinos-menos" ( $v_{-}$) oriundos do decaimento $\beta$ (conhecido, na época, como $\beta$ - e que era o elétron) produzido pelo reator do Hanford Engineering Works (HEW), situado em Washington, com prótons de um cintilador líquido, em uma reação nuclear do tipo: $v_{-}+\mathrm{p} \rightarrow \mathrm{n}$ $+\beta^{+}$, com $\beta^{+}$representando o pósitron $\left(\mathrm{e}^{+}\right)$. Estes, por sua vez, ao encontrarem elétrons livres do fluido do cintilador, se aniquilavam e se transformavam em fótons, responsáveis, portanto pela cintilação. O resultado dessa experiência foi apresentado por Reines e Cowan, em $1953^{38}$.

Ressalte-se que, em sua experiência (C $\ell$-Ar) de 1951, Davis Junior observou que o BGRR/BNL não era uma forte fonte de $\bar{v}$ para realizar a reação $\mathrm{C} \ell$-Ar, e considerando o sucesso de Reines e Cowan com o HEW, em 1954, Davis Junior pensou em utilizar o Savannah River Nuclear Reactor (SRNR), localizado na Carolina do Sul e que, naquele momento, era a fonte mais intensa de $v$. Desse modo, na base deste reator, ele voltou a usar o tanque de 3.800 litros contendo $\mathrm{CC}_{4}$ e, como no caso da experiência realizada no BGRR/BNL Davis Junior não conseguiu mostrar a identidade neutrino-antineutrino, pois encontrou que as taxas de captura de $v$ e de $\bar{v}$ por parte do $\mathrm{C} \ell$, eram diferentes, numa relação de $1 / 5$. Em vista disso, em 195539, Davis publicou um artigo no qual discutiu apenas a possibilidade de detectar antineutrinos oriundos de reatores por intermédio da reação C $\ell$-Ar. Observe-se que, em $1956^{40}$ e em 196041, Cowan, Reines, F. B. Harrison, H. W. Kruse e A. D. McGuire confirmaram o resultado da experiência de 1953, usando um tanque de $1 / 2 \mathrm{~m}^{3}$ de água para detectar o seu $v_{-}$proveniente do SRNR. Note-se que, em 195842, Davis Junior voltou a usar o SRNR, usando um tanque de $11.400 \ell$ contendo $\mathrm{CC} \ell_{4} \mathrm{e}$, desta vez, encontrou uma relação de $1 / 20$ entre as taxas de neutrino/antineutrino.

Destaque-se que, em 195743, o físico paquistanês Abdus Salam (1926-1996; PNF, 1979 ) aventou a hipótese de o neutrino eletrônico $\left(v_{\mathrm{e}}\right)$ possuir massa em seu artigo sobre a quebra da paridade na interação fraca, confirmando essa quebra descoberta, em 195644, pelos físicos sino-norte-americanos Tsung-Dao Lee (n.1926; PNF, 1957) e Chen Ning Yang (n.1925; PNF, 1957). É oportuno registrar que, em 197145, o físico brasileiro Mario Novello (n.1942), propôs que um mecanismo gravitacional era capaz de gerar a massa dos neutrinos, bem como

\footnotetext{
38 Physical Review, v. 92, p. 830, 1953.

${ }^{39}$ Physical Review, v. 97, p. 766, 1955.

40 Science, v. 124, p. 103, 1956.

41 Physical Review, v. 117, p. 159, 1960.

42 UNESCO Conference, Paris 1, p. 728, 1958.

43 Nuovo Cimento, v. 5, p. 299, 1957.

${ }^{44}$ Physical Review, v. 102, p. 290; 104, p. 254, 1956.

45 Lettere al Nuovo Cimento, v. 1, p. 252, 1971.
} 
a de outros férmions ${ }^{46}$.

Os dois neutrinos [inicialmente associados ao elétron $\left(v_{\mathrm{e}}\right)$ e ao múon $\left(v_{\mu}\right)$ ] previstos pelo TP-W-T foram deduzidos em experiências independentes conduzidas, em 1957, pelos físicos norte-americanos Richard Lawrence Garwin (n.1928), Leon Max Lederman (n.1922; PNF, 1988) e Marcel Weinrich (1927-2008)47 e pelos físicos, o norte-americano Jerome Isaac Friedman (n.1930; PNF, 1990) e o suíço Valentine Louis Teledgi (n.1922)48. Por sua vez, em 1960, Pontecorvo e o físico norte-americano Melvin Schwartz (1932-2006; PNF, 1988) ${ }^{49}$ e, independentemente, Lee e Yang ${ }^{50}$ propuseram um tipo de experiência para a produção de $v_{\mu}$ em decorrência do decaimento do $\pi^{+}$, ou seja: $p+p \rightarrow p+n+\pi^{+}\left(\rightarrow \mu++v_{\mu}\right)$. De posse desses neutrinos, Schwartz e os físicos norte-americanos Lederman, Jack Steinberger (n.1921; PNF, 1988), Gordon Danby, Jean-Marc Gaillard, Konstantin Goulianos e Nariman B. Mistry, realizaram uma experiência no ciclotron Nevis do BNL, em 196251, na qual comprovaram a existência do $v_{\mu}$, bem como confirmaram a existência dos neutrinos/antineutrinos associados ao elétron $\left(v_{\mathrm{e}}-/+\right)$ e ao múon $\left(v_{\mu^{-}} /+\right)$, em reações dos tipos: $\mathrm{p}+v_{\mathrm{e}}+\rightarrow \mathrm{n}+\mathrm{e}^{+} ; \mathrm{n}+\mathrm{v}_{\mathrm{e}^{-}} \rightarrow \mathrm{p}+\mathrm{e}^{-} ; \mathrm{p}+$ $v_{\mu}+\rightarrow \mathrm{n}+\mu^{+}$e $\mathrm{n}+v_{\mu^{-}} \rightarrow \mathrm{p}+v_{\mu^{-}}$. É oportuno salientar que a existência de $v_{\mathrm{e}^{-}} /+$e $v_{\mu^{-}} /+$foi confirmada no CERN, em 196452, em experiências, envolvendo 37 cientistas (físicos e engenheiros), dentre os quais o físico brasileiro Roberto Aureliano Salmeron (n.1922).

Por fim, vejamos a descoberta do terceiro neutrino. Para entendê-lo, façamos um pequeno resumo histórico. Como vimos acima, durante as décadas de 1930, 1940, 1950 e 1960, várias partículas elementares (carregadas e neutras) foram descobertas. Em vista disso, foi necessário fazer uma classificação delas e, também, formular um Modelo Matemático para entender a sua Dinâmica, o hoje conhecido Modelo Padrão da Física das Partículas Elementares (MPFPE), desenvolvido entre 1970 e 1973. Assim, de um modo geral, as partículas elementares constituintes da matéria classificam-se em: hádrons e léptons. Os hádrons (do grego adros, que significa "grosso e volumoso"), nome cunhado pelo físico russo Lev Borisovich Okun (n.1929), em 196253, são partículas que sofrem os quatro tipos de força (interação): gravitacional, eletromagnética, fraca e forte, e são de dois tipos: bárions e mésons. Os bárions (do grego barys, que significa "pesado"), cujo nome foi cunhado pelo físico e historiador da ciência, o holandês-

\footnotetext{
46 NOVEllo, M. A massa do neutrino. Scientific American Brasil, v. 163, p. 21, dezembro de 2015; e Os Cientistas da Minha Geração. Livraria da Física, 2016; BASSALO, J. M.; CARUSO, F. Salam. Livraria da Física, 2016.
}

${ }^{47}$ Physical Review, v. 105, p. 1415, 1957.

48 Physical Review, v. 105, p. 1681, 1957.

${ }^{49}$ Soviet Physics - JETP 10, p. 1236, 1960.

50 Physical Review Letters, v. 4, p. 306; 307, 1960.

${ }^{51}$ Physical Review Letters, v. 9, p. 36, 1962.

52 Physics Letters, v. 12, p. 281; 13, p. 80; 86, 1964.

53 Procedings of the Internacional Conference on High Energy Physics, CERN, p. 845, 1962. 
norte-americano Abraham Pais (1918-2000), em 195454, são férmions [que satisfazem a Estatística de Fermi-Dirac (1926)], formados por três quarks [introduzidos pelo físico norte-americano Murray Gell-Mann (n.1929; PNF, 1969), em 196455 e se juntam em duas categorias: nucleóns e hyperons. O termo nucleón (p, n) foi introduzido em 1941, por Möller, como já frisamos, e os hyperons (do grego iper, que significa "super", "acima" ou "além de"), termo introduzido pelo físico francês Louis Leprince-Ringuet (1901-2000), em 195356, representam as partículas com massa intermediária entre nucleóns e píons. Note-se que, por essa ocasião, ainda não se sabia que os píons tinham massa inferior à dos nucleóns que estes também tinham massa inferior à dos hyperons. Por sua vez, os mésons [do grego meso, que significa "médio" e que receberam essa denominação, em 1939, em trabalhos independentes, dos físicos, o inglês Charles Galton Darwin (1887-1962) ${ }^{57}$ e o indiano Homi Jehangir Bhabha (1909-1966) ${ }^{58}$, são bósons [que satisfazem a Estatística de Bose-Einstein (1924)], formados por um par quark/antiquark. Os léptons (do grego leptos, que significa "fino" ou "pequeno"), são férmions e não sofrem interação forte, e tiveram seu nome cunhado por Möller e Pais, em 1946, para representar qualquer partícula de massa pequena como o elétron e o neutrino ${ }^{59}$.

Nesta oportunidade, é interessante pausar a história da descoberta do terceiro neutrino e fazer uma observação sobre as leis de conservação (que decorrem do MPFPE) que regem as reações nucleares. Além da conservação da energia, do momento linear e do momento angular, oriundos da Física Clássica, a descoberta das partículas elementares levou à introdução de novas leis de conservação, das quais destacaremos duas: 1) Lei de Conservação do número bariônico $(\mathrm{B})$ e vale $(\mathrm{B}=+1)$ para os bárions e $(\mathrm{B}=-1)$ para os antibárions, e $\mathrm{B}=0$, para as demais partículas; 2) Lei de Conservação do número leptônico $(\mathrm{L})$ e vale $(\mathrm{L}=+1)$ para os léptons e $(\mathrm{L}=-1)$ para os antiléptons, $\mathrm{e} \mathrm{L}=0$, para as demais partículas. É por causa delas que se explicam as seguintes reações dadas por: $v_{\mathrm{e}}+\mathrm{n} \leftrightarrow \mathrm{p}+\mathrm{e}^{-} \mathrm{e} \bar{v}_{\mathrm{e}}+\mathrm{p} \rightarrow \mathrm{n}+\mathrm{e}^{+}$, pois $\mathrm{p}$ e $\mathrm{n}$ são bárions $(\mathrm{B}=+1)$, $\mathrm{e}^{-} \mathrm{e} v_{\mathrm{e}}$ são léptons $(\mathrm{L}=+1)$, e $\mathrm{e}^{+} \mathrm{e} \bar{v}_{\mathrm{e}}$ são antiléptons $(\mathrm{L}=-1)$.

Continuemos com a história da descoberta do terceiro neutrino. Como destacamos acima, na primeira metade da década de 1970, só se conheciam dois tipos de léptons [eletrônico $\left(\mathrm{e}^{-,+}\right)$e muônico $\left(\mu^{-,+}\right)$, com seus respectivos neutrinos/antineutrinos $\left.\left(v_{\mathrm{e}}, v_{\mu}, \bar{v}_{\mathrm{e}}, \bar{v}_{\mu}\right)\right]$. Contudo, o MPFPE previa a existência de mais um lépton/antilépton e seu respectivo neutrino/antineutrino, uma vez que o trabalho de Gell-Mann, de 1964 (referido acima), previa e existência de três quarks. Desse modo, se iniciou a busca do terceiro lépton, e seu companheiro neutrino.

\footnotetext{
${ }^{54}$ Procedings of the Internacional Conference on Theoretical Physics, Kyoto, p. 157, 1954.

55 Physics Letters, b. 8, p. 214, 1964.

56 Annual Review of Nuclear Science, v. 3, p. 39, 1953.

${ }^{57}$ Nature, v. 143, p. 276, 1939.

58 Nature, v. 143, p. 602, 1939.

59 PAIS, A. Inward Bound: of matter and forces in the physical world. Oxford University Press, 1995.
} 
Assim, em outubro de $1975^{60}$, uma equipe de pesquisadores do SLAC, sob a liderança do físico norte-americano Martin Lewis Perl (n.1927; PNF, 1995), realizou uma experiência na qual estudaram a colisão elétron-pósitron $\left(\mathrm{e}^{-}-\mathrm{e}^{+}\right)$, com a produção anômala de elétrons (e) e de múons $(\mu)$. Essa produção decorria do decaimento de uma nova partícula, a princípio denominada de $\mathrm{U}$, inicial da palavra unknow ("desconhecido", em inglês), decorrente da reação: $\mathrm{e}^{-}+\mathrm{e}^{+} \rightarrow \mathrm{U}^{-}$ $+\mathrm{U}^{+}$, com o seguinte modo de decaimento de $\mathrm{U}: \mathrm{U} \rightarrow \mu(\mathrm{e})+\bar{v}_{\mu}\left(\bar{v}_{e}\right)+v_{U}$. Ainda em 1975, Perl e seu estudante de pós-graduação, o físico grego Petros Afentoulis Rapidis (n.1951), sugeriram o nome $\operatorname{tau}(\tau)$, inicial da palavra $\tau \rho \iota \tau o v$ ("triton", terceiro em grego), para esse terceiro lépton ("pesado"), com a massa aproximada entre 1,6 e 2,0 GeV/c ${ }^{2}\left(1 \mathrm{GeV}=10^{3} \mathrm{MeV}\right)^{61}$. Essa partícula foi comprovada por Perl [e mais 36 físicos, dentre eles Rapidis e o norte-americano Burton Richter (n.1931; PNF, 1976)], em 197662.

Hoje, existem o $\tau^{+}$e sua antipartícula $\tau^{-}$, com a massa aproximada de $1,77 \mathrm{GeV} / \mathrm{c}^{2}$, bem como seus respectivos neutrinos $\left(v_{\tau}\right)$ e antineutrinos (anti- $v_{\tau}$ ), de acordo com o MPFPE, segundo o qual, em qualquer membro de uma reação nuclear, todo lépton (antilépton) é acompanhado de seu antineutrino (neutrino) correspondente, porém, ainda segundo o MPFPE, eles não possuem massa.

Em seguida, trataremos da descoberta dos neutrinos solares e ver como foram observadas suas oscilações. Segundo anotamos acima, no artigo de 1955, Davis Junior examinou a possibilidade de detectar antineutrinos oriundos de reatores por intermédio da reação $\mathrm{C} \ell$-Ar, bem como a possibilidade de construir detectores para medir o fluxo de neutrinos solares liberados nos CPP e CCNO. Mais tarde, em $1958^{63}$, H. D. Holmgren e R. L. Johnston investigaram a produção de neutrinos em reações nucleares do tipo: ${ }_{2} \mathrm{He}^{3}+{ }_{2} \mathrm{He}^{4} \rightarrow{ }_{4} \mathrm{Be}^{7}+\gamma$ e, também, do tipo: $\mathrm{e}^{-}+{ }_{4} \mathrm{Be}^{7} \rightarrow{ }_{3} \mathrm{Li}^{7}+v_{\text {e. }}$ Ainda em 1958, em trabalhos independentes, os físicos norte-americanos Alastair Graham Walter Cameron (1925-2005) ${ }^{64}$ e William Alfred Fowler (1911-1995; PNF, 1983) ${ }^{65}$ conjecturaram que o processo de geração de energia nas estrelas do tipo do Sol (solares) deve-se a reações nucleares do tipo: ${ }_{4} \mathrm{Be}^{7}+p \rightarrow{ }_{5} \mathrm{~B}^{8}+\gamma$, seguida de: ${ }_{5} \mathrm{~B}^{8} \rightarrow{ }_{4} \mathrm{Be}^{8}+\mathrm{e}^{+}$ $+v_{\text {e }}$ Como nesses artigos havia a possibilidade de usar os $v_{e}$ para produzir a reação $\mathrm{C} \ell$-Ar, Davis Junior construiu um tanque de $3.800 \ell$ de percloroetileno $\left(\mathrm{C}_{2} \mathrm{C}_{4}\right)$ na Barberton Limestone Mine, em Ohio. Contudo, não houve nenhum sinal de ve produzido pelo Ar.

Continuemos com a busca dos neutrinos solares (e suas oscilações). Um dos primeiros

\footnotetext{
60 Physical Review Letters, v. 35, p. 1489, 1975.

61 PERL, M. L. Reflections on the Discovery of the Tau Lepton. Nobel Lecture, 08 de dezembro de 1995.

62 Physics Letters, B63, p. 466, 1976.

63 Physical Review, v. 113, p. 1556), 1958.

64 Bulletin of the American Physical Society, v. 3, p. 227, 1958; Annual Review of Nuclear Science, v. 8, p. 299, 1958.

65 Astrophysical Journal, v. 127, p. 551, 1958.
} 
modelos sobre a produção da energia estelar foi apresentado, em 1958, pelo físico alemão Martin Schwarzschild (1912-1997) em seu livro intitulado Structure and Evolution of the Stars66. Posteriormente, a partir de 1962, o astrofísico norte-americano John Norris Bahcall (19342005) desenvolveu o Modelo Padrão Solar [Standard Solar Model (SSM)] sobre o fluxo de neutrinos solares (NS) ao demonstrar que a taxa de emissão de $v_{\mathrm{e}}$ pelo boro- $8\left({ }_{5} \mathrm{~B}^{8}\right)$ era 20 vezes mais alta do que o valor previamente esperado, com energia máxima de 14,06 MeV. Em vista disso, Davis Junior planejou uma experiência do tipo $\mathrm{C} \ell$-Ar e, depois de trocar cartas com J. N. Bahcall resolveram publicar seus resultados, em 1964: o teórico de Bahcall ${ }^{67}$ e o experimental de Davis Junior ${ }^{68}$. Contudo, para que a experiência tivesse sucesso, era necessário que o tanque de $\mathrm{C}_{2} \mathrm{C}_{4}$ fosse bem enterrado.

Desse modo e com essa ideia em mente e ainda contando com o suporte do BNL e do Council of Economic Advisers dos Estados Unidos (CEA/US), Davis Junior iniciou, em 1965, o planejamento de uma experiência do tipo $\mathrm{C} \ell$-Ar, usando um tanque de $378.000 \ell$ de $_{2} \mathrm{C}_{4} \mathrm{e}$ que foi enterrado na Homestake Gold Mine, em Lead, Dakota do Sul, a uma profundidade de $1.478 \mathrm{~m}$ e este experimento se tornou operacional em 1967. Os primeiros resultados dessa experiência foram publicados em $1968^{69}$, no artigo assinado por Davis Junior, D. S. Harmer e K. C. Koffman, no qual relataram que haviam encontrado um limite superior para o fluxo de $v_{\mathrm{e}}$ bem abaixo do previsto, ou seja: 3 SNU [1 Solar Neutrino Unit $(\mathrm{SNU})=10-36$ capturas por segundo e por núcleo absorsor], uma vez que, ainda em 196870, J. N. Bahcall, Nata A. Bahcall e G. Shaviv demonstraram, usando o SSM, que aquele limite era de: (7,5 \pm 3) SNU. É interessante registrar que, em 197371, A. J. R. Prentice explicou que os NS eram devidos às inhomogeneidades da composição do Sol; por exemplo, enquanto o hidrogênio $(\mathrm{H})$ era queimado em suas partes mais externas, o caroço solar era composto de hélio (He). Por sua vez, também, em 197372 , P. Demarque, J. G. Mengel e A. V. Sweigart sugeriram que os NS estavam relacionados com o fato de que o interior do Sol gira mais rápido que o seu exterior.

Em 1984, Davis Junior deixou o BNL e transferiu o Experimento Homestake para a Universidade da Pensilvânia, e continuou a realizar trabalhos, com novos colaboradores. Assim, em 1987, Davis Junior organizou uma colaboração com físicos soviéticos, conhecida como Soviet-American Gallium Experiment (SAGE), com o objetivo da medição rádioquímica do fluxo de neutrino eletrônico solar baseada na seguinte reação $v_{\mathrm{e}}+{ }_{31} \mathrm{Ga}^{71} \rightarrow \mathrm{e}^{-}+{ }_{32} \mathrm{Ge}^{71}$ (lembrar que: $\left.v_{\mathrm{e}}+\mathrm{n} \rightarrow \mathrm{p}+\mathrm{e}^{-}\right)$, na qual a presença do neutrino eletrônico solar $\left(v_{\mathrm{e}}\right)$ é evidenciada pelo

\footnotetext{
66 Princeton University Press.

67 Physical Review Letters, v. 12, p. 300, 1964.

68 Physical Review Letters, v. 12, p. 303, 1964.

${ }^{69}$ Physical Review Letters, v. 20, 1205, 1968.

${ }^{70}$ Physical Review Letters, v. 20, 1209, 1968.

${ }^{71}$ Monthly Notices of Royal Astronomical Society, v. 163, p. 331, 1973.

72 Nature, v. 246, p. 33, 1973.
} 
decaimento do germânio radioativo $\left({ }_{32} \mathrm{Ge}^{71}\right)$, que tem uma vida média de 11,43 dias. $\mathrm{O}$ alvo para essa reação era um tanque contendo 50-57 toneladas (t) do líquido metálico de gálio $(\mathrm{Ga})$ enterrado no Baksan Neutrino Observatory (BNO), nas montanhas do Cáucaso, na Rússia. Desse modo, em 199173, Davis Junior e seus 27 colaboradores do SAGE apresentaram seus primeiros resultados. Novos resultados foram publicados, em 199974, por Davis Junior e 24 colaboradores. Desde que o SAGE funcionou, de janeiro de 1990 até dezembro de 2007, foram realizados 168 experimentos, cujos resultados apresentavam a mesma discrepância (cerca de $1 / 3$ ) entre os valores medidos do fluxo de $v_{\mathrm{e}}$ e os previstos pelo SSM desenvolvido por J. N. Bahcall, desde 1962 (como vimos acima), modelo esse que foi refinado nos seguintes trabalhos: J. N. Bahcall, Nicola Cabibbo (1935-2010) e A. Yahil75; J. N. Bahcall, W. F. Huebner, S. H. Lubow, P. D. Parker e R. K. Ulrich ${ }^{76}$; J. N. Bahcall e o físico norte-americano Sheldon Lee Glashow (n.1932; PNF, 1979) ${ }^{77}$; J. N. Bahcall, W. D. Arnett, R. P. Kirshner e S. E. Woosley ${ }^{78}$; J. N. Bahcall, Neutrino Astrophysics ${ }^{79}$; J. N. Bahcall, K. Lande, R. E. Lanou, J. G. Learned, R. G. H. Robertson e Lincoln Wolfenstein ${ }^{80}$; J. N. Bahcall, M. H. Pinsonneault, S. Basu e J. Christensen-Dalsgaard ${ }^{81}$; J. N. Bahcall ${ }^{82}$; J. N. Bahcall, Plamen I. Krastev e o físico russo Alexei Yuryevich Smirnov (n.1951) ${ }^{83}$; J. N. Bahcall, Krastev e Smirnov84; Bahcall, Pinsonneault e $\mathrm{Basu}^{85}$. É interessante destacar que, além do Ga, o SAGE usou também o cromo $\left({ }_{24} \mathrm{Cr}^{51}\right)$ como fonte de neutrinos, cuja energia do fluxo é similar a dos neutrinos solares do ${ }_{4} \mathrm{Be}^{7}$, e que os resultados dessas experiências, realizadas entre 2002-2007, podem ser vistos nos artigos publicados em 2009: Physical Review, C80, n. 015807; arXiv: 0901.2200 (en.wikipedia.org/wiki/SAGE).

Paralelamente a esse trabalho no SAGE, Davis Junior continuou com suas experiências no Homestake. Com efeito, em 1998 ${ }^{86}$, Davis Junior e mais 07 colaboradores mediram o

\footnotetext{
73 Physical Review Letters, v. 67, p. 3332, 1991.

74 Physical Review Letters, v. 83, p. 4686, 1999.

75 Physical Review Letters, v. 28, p. 316, 1972.

76 Reviews of Modern Physics, v. 54, p. 767, 1982.

77 Nature, v. 326, p. 476, 1987.

78 Annual Review of Astronomy and Astrophysics, v. 27, p. 629, 1989.

79 World Scientific, 1989.

${ }^{80}$ Nature, v. 375, p. 29, 1995.

81 Physical Review Letters, v. 78, p. 171, 1997.

82 Physical Review, C56, p. 3391, 1997.

83 Physical Review, D58, n. 096016, 1998.

${ }^{84}$ Physical Review, D60, n. 093001, 1999.

85 Astrophysical Journal, v. 555, p. 990, 2001.

86 Astrophysical Journal, v. 201, p. 505, 1998.
} 
fluxo de neutrinos solares usando a reação $\mathrm{C} \ell$-Ar e o detector de $\mathrm{C}_{2} \mathrm{C} \ell_{4}{ }^{87}$.

É oportuno ressaltar que a discrepância de 1/3, entre os valores medidos de fluxos de $v_{\mathrm{e}}$ e os previstos pelo SSM, também foi encontrada em experiências realizadas pelo Gallium Experiment (GALLEX) e pelo seu sucessor, o Gallium Neutrino Observatory (GNO), das quais trataremos mais adiante. Esses experimentos foram idealizados pelo Laboratori Nazionali del Gran Sasso (LNGS) [criado pelo Istituto Nazionale di Fisica Nucleare (INFN)] e localizados na montanha de Gran Sasso, de 2.912 m de altura, na província de Abruzzo, da cidade de L'Aquila, Itália, para detectar neutrinos solares de baixa energia [com um limite superior de 233,2 $\left.\mathrm{keV}\left(1 \mathrm{keV}=10^{3} \mathrm{eV}\right)\right]$. Note-se que essa colaboração internacional usou um tanque de $54 \mathrm{~m}^{3}$ cheio de $101 \mathrm{t}$ de uma solução ácida de triclorido-hydroclórico $\left(\mathrm{Cl}_{3} \mathrm{HCl}\right)$, contendo 30,3 t de Ga. Nesses experimentos, também foi possível detectar neutrinos solares do ciclo CPP, com um limite superior de $0,420 \mathrm{MeV}$.

Durante o período (1971-1997) em que o GALLEX funcionou, seu detector mediu uma taxa total de 77,5 SNU, com um decaimento médio diário de 0,75. Das experiências realizadas por essa colaboração internacional de físicos, destaquemos as de $1992^{88}$, realizadas por 54 físicos [dentre eles, o físico alemão Rudolf Ludwig Mössbauer (n.1929; PNF, 1961)]; e o de $1999^{89}$, conduzidas por 49 físicos (incluindo Mössbauer).

Por sua vez, em abril de 1998, começou o experimento GNO (com $30 \mathrm{t}$ de Ga e tendo ainda o Ge como fonte de $v_{\mathrm{e}}$ ), também liderado pelo LNGS, por intermédio do BORONSOLAR NEUTRINO EXPERIMENT (BOREX). No período de seu funcionamento, maio de 1998-janeiro de 2002, seus resultados foram apresentados em 200090, por 30 cientistas; e na Neutrino 2002 Conference, realizada em Munique, na Alemanha. Em resumo, nas experiências realizadas pelas colaborações GALLEX/GNO, os valores encontrados foram: GALLEX: 77,5 \pm 62 (estatístico) + 4,3/-4.7 (sistemático) SNU, e GNO: 65,2 \pm 6,2 (estatístico) \pm 3.0 (sistemático) SNU, comparado ao valor teórico de 138 SNU (www.lngs.it). Registre-se que essa discrepância foi resolvida com novos experimentos e levando em conta a oscilação de neutrinos solares, no Sudbury Neutrino Observatory (SNO), localizado na Inco Nickel Mine, em Sudbury (Ontário, Canadá), sob a direção McDonald (desde 1990) e apresentado em 2001 e 2002, segundo veremos mais adiante ${ }^{91}$.

Cremos ser oportuno falar algo sobre o BOREX. Em maio de 2007, foi criada a $B O$ REXINO (diminutivo italiano de Borex) Collaboration (uma colaboração internacional com a participação de cientistas de vários países: Alemanha, Canadá, Estados Unidos, França, Itália,

\footnotetext{
87 Mais detalhes do trabalho de Davis Junior, ver sua Nobel Lecture: A Half-Century with Solar Neutrinos (08 de dezembro de 2002; e-Nobel Museum).

88 Physics Letters, B285, p. 376, 1992.

${ }^{89}$ Physics Letters, B447, p. 127, 1999.

${ }^{90}$ Physics Letters, B447, p. 16, 2000.

91 <en.wikipedia.org/wiki/GALLEX>.
} 
Polônia, Rússia e Ucrânia), usando um detector líquido de cintilação e localizado na montanha italiana de Gran Sasso, objetivando detectar antineutrinos/neutrinos radioativos do interior e da crosta de nosso planeta Terra (geoneutrinos/geoantineutrinos ou geológicos) e antineutrinos/neutrinos solares. Os principais resultados dessa Colaboração foram: 1) em 200792 (77 cientistas) e $2008^{93}$ (133 cientistas), detectados os primeiros neutrinos/antineutrinos solares produzidos pelo berílio radioativo $\left({ }_{4} \mathrm{Be}^{7}\right) ; 2$ ) em $2010^{94}$ (88 cientistas), detectados os primeiros geoantineutrinos dos elementos radioativos e, dentre eles: urânio $\left({ }_{92} \mathrm{U}^{238}\right)$, tório $\left({ }_{90} \mathrm{Th}^{232}\right)$ e potássio $\left({ }_{19} \mathrm{~K}^{40}\right)$; 3) em $2011^{95}$ (89 cientistas) e (91 cientistas) ${ }^{96}$, respectivamente, confirmação de neutrinos/antineutrinos solares emitidos pelo ${ }_{4} \mathrm{Be}^{7}$ e pela fusão nuclear (próton-próton) no centro do Sol; 4) em $2012^{97}$ (105 cientistas), foi medida a velocidade do neutrino muônico $\left(v_{\mu}\right)$ produzido no CERN (sobre essa medida é interessante destacar que, inicialmente, ela apresentava um valor > c, e logo depois foi encontrado ser compatível com c); 5) em 201398 (93 cientistas), novos geoneutrinos/geoantineutrinos foram detectados: em 201499 (91 cientistas), foi confirmada a fusão nuclear (próton-próton) no centro do Sol; e, em agosto de $2015{ }^{100}$ (102 cientistas) foi divulgada uma espectroscopia de geoneutrinos/geoantineutrinos. (en.wikipedia.org/BOREXINO).

Continuemos com o histórico sobre o tema do PNF2015, agora tratando das oscilações de neutrinos $\left(v_{\mathrm{e}} \rightarrow v_{\mu} \rightarrow v_{\tau}\right.$ ). As primeiras considerações sobre a oscilação de neutrinos (antineutrinos) eletrônicos solares $\left(v_{\mathrm{e}} \leftrightarrow \bar{v}_{\mathrm{e}}\right)$ no vácuo foi aventada por Pontecorvo, em $1957101 \mathrm{e}$ em 1958 ${ }^{102}$. Destaque-se que, com a descoberta do $v_{\mu}$, em 1962 (já registrada), os físicos japoneses Z. Maki, M. Nakagawa e Shoichi Sakata (1911-1970), ainda em 1962103, discutiram a possibilidade de esses dois conhecidos "sabores" de neutrinos solares serem uma mistura de dois auto-estados de massa dos mesmos. Contudo, o primeiro modelo fenomenológico da oscilação de neutrinos (eletrônicos e muônicos) solares $\left(v_{\mathrm{e}} \leftrightarrow v_{\mu}\right)$ foi proposto por Pontecorvo, em

\footnotetext{
${ }^{92}$ Physics Letters, B658, p. 101, 2007.

${ }^{93}$ Physical Review Letters, v. 101, n. 091302, 2008.

${ }^{94}$ Physics Letters, B687, p. 299, 2010.

95 Physical Review Letters, v. 107, n. 141302, 2011.

${ }^{96}$ Physical Review Letters, v. 108, n. 051302, 2011.

97 Physics Letters, B716, p. 401, 2012.

${ }^{98}$ Physics Letters, B722, p. 29, 2013.

${ }^{99}$ Nature, v. 512, p. 383, 2014.

100 Physical Review, D92, n. 031101, 2015.

101 Soviet Physics, JETP 6, p. 984, 1957.

102 Soviet Physics, JETP 7, p. 172, 1958.

103 Progress in Theoretical Physics, v. 28, p. 870, 1962.
} 
$1968^{104}$ e melhorado por ele e pelo físico russo físico russo Vladimir Naumovich Gribov (19301997), em 1969105. É interessante destacar que, no túmulo de Pontecorvo, em Roma, está escrita a seguinte equação: $v_{\mu} \neq v_{\mathrm{e}}$. (Close, op. cit.).

Por sua vez, a possibilidade da existência do neutrino tauônico $\left(v_{\tau}\right)$, decorrente das experiências de Perl (1975-1976) que analisamos acima, levou o FERMILAB, por intermédio de seu acelerador Tevatron [acelerador de prótons em um anel de 6,3 km e energia de até $1 \mathrm{TeV}$ ( $\left.=10^{3} \mathrm{MeV}\right)$, com o início de operação ocorrido em 1983], a montar um experimento (Collaboration) [Direct Observation of the NU Tau, E872 (DONUT)], em 1997, para observar a interação de $v_{\tau}$, anunciada em 20 de julho de 2000 e publicada em 2001 ${ }^{106}$. É oportuno registrar que o $v_{\tau}$ foi confirmado, em 2012, em experiências realizadas no Large Hadron Collider (LHC/CERN), por duas Colaborações: A Toroidal LHC ApparatuS (ATLAS) e Compact Muon Solenoid (CMS). Na do ATLAS ${ }^{107}$, participaram quase 3.000 cientistas, sendo treze (13) cientistas brasileiros; na do $\mathrm{CMS}^{108}$ com também quase 3.000 cientistas, dentre eles trinta e cinco (35) brasileiros ${ }^{109}$.

Os trabalhos de Pontecorvo, Maki, Nakagawa e Sakata [e que foram confirmados pelos físicos russos S. P. Mihkeev e Smirnov, em 1985110, em 1986111] ensejaram o desenvolvimento de um modelo para explicar as oscilações de neutrinos $(\mathrm{ON})$ [de qualquer "sabor" e massivos (embora contra o MPFPE, pois para este, o neutrino não possui massa, como já destacamos): eletrônico $\left(v_{\mathrm{e}}\right)$, muônico $\left(v_{\mu}\right)$ e tauônico $\left.\left(v_{\tau}\right)\right]$. Desse modo, hoje, a ON é devido a uma mistura dos auto-estados do Hamiltoniano $(\mathrm{H})$ e dos auto-estados da interação fraca envolvendo seus léptons correspondentes $(\mathrm{e}, \mu, \tau)$. Assim, quando os neutrinos se propagam através do espaço, os fatores de fase correspondentes aos auto-estados oscilam devido a diferenças de massa dos auto-estados de H. Note-se que o conceito de mistura de neutrinos é o resultado natural de uma Teoria de 'Gauge' com neutrinos (de três "sabores") massivos, cuja transformação unitária relacionando os auto-estados de massa e de "sabor", sendo estes dados por: $\left|v_{\alpha}>=\Sigma_{\mathrm{i}} \mathrm{U}_{\alpha \mathrm{i}}\right| v_{\mathrm{i}}$ $>$ e $\left|v_{\mathrm{i}}>=\Sigma_{\alpha} \mathrm{U}_{\alpha \mathrm{i}}^{*}\right| v_{\alpha}>$, onde $\mid v_{\alpha}>$ corresponde a um neutrino com "sabor" bem definido $\left(v_{\mathrm{e}}, v_{\mu}, v_{\tau}\right.$ ou $\left.\bar{v}_{\mathrm{e}}, \bar{v}_{\mu}, \bar{v}_{\tau}\right)$ e $\mid v_{\mathrm{i}}>$ a um neutrino de massa mi definida $\left[\mathrm{i}=1\left(v_{\mathrm{e}}\right), 2\left(v_{\mu}\right), 3\left(v_{\tau}\right)\right]$, $\mathrm{U}_{\text {ai }}$ é a matriz de Pontecorvo-Maki-Nakagawa-Sakata (PMNS) e (*) significa a matriz complexa conjugada. É interessante ressaltar que, com essa matriz, se calcula a probabilidade de oscilação de dois neutrinos: $\mathrm{P}_{\mathrm{i} \rightarrow \mathrm{j},(\mathrm{i} \neq \mathrm{j})} \approx \operatorname{sen}^{2}\left(2 \theta_{\mathrm{ij}}\right)=\operatorname{sen}^{2}\left[1,27\left(\Delta \mathrm{m}^{2} \mathrm{ij} / \mathrm{L}\right)\right]$, sendo $\theta_{\mathrm{ij}}$ (o ângulo

\footnotetext{
${ }^{104}$ Soviet Physics, JETP 26, p. 172, 1968.

105 Physics Letters, B28, p. 493, 1969.

106 Physics Letters, B504, p. 218, 2001.

107 Physics Letters, B716, p. 1, 2012.

108 Physics Letters, B716, p. 30, 2012.

109 <www.searadaciencia.ufc.br/bassalo/PNF2013>.

110 Yadernaya Fizika, v. 42, p. 1441, 1985.

111 Nuovo Cimento, C9, p. 17, 1986.
} 
de mistura), $\Delta \mathrm{m}_{\mathrm{ij}}^{2}=\mathrm{m}_{\mathrm{i}}^{2}-\mathrm{m}_{\mathrm{j}}^{2}\left[\mathrm{~m}_{\mathrm{i}}\left(\mathrm{ou} \mathrm{m}_{\mathrm{j}}\right)\right]$ medido em eV, $\mathrm{L}$ (distância entre a fonte emissora e o detector de neutrinos) considerado em km e E (energia do feixe de neutrinos suposta constante), tomada em GeV. Ressalve-se que as experiências com neutrinos envolvem o cálculo de $\Delta \mathrm{m}^{2}{ }_{\mathrm{ij}}$ e $\theta_{\mathrm{ij}}$, segundo veremos mais adiante ${ }^{112}$.

Agora, passemos a analisar os trabalhos dos nobelistas Kajita e McDonald. Kajita foi para o ICRR/UT trabalhar com Koshiba, que estudava o espalhamento de neutrinos/antineutri$n o s\left(v_{\mathrm{e}} / \bar{v}_{e}\right)$ [decorrentes do decaimento de nucleóns ( $\mathrm{p}$ e $\left.\mathrm{n}\right)$ ] pelos elétrons $\left(\mathrm{e}^{-}\right)$e por prótons (p) da água, bem como o decaimento do $\mathrm{p}$. Por exemplo, no caso do espalhamento $v_{\mathrm{e}}-\mathrm{e}^{-}$, como a massa do e é de $0,5 \mathrm{MeV}$ (no sistema no qual se admite $\mathrm{c}=1$ ) e considerando que os $v_{\mathrm{e}}$ têm energia muito mais alta (p.e.: $10 \mathrm{MeV}$ ), então, na colisão entre eles, o e- recua praticamente na direção da incidência do $v_{\mathrm{e}} \mathrm{e}$, portanto, há uma relação direta entre os espectros energéticos dessas duas partículas. O tempo dessa colisão é da $\sim 10 \mathrm{~ns}\left(1 \mathrm{~ns}=10^{-9} \mathrm{~s}\right)$. Para esse estudo, Koshiba usava a Radiação de Vavilov-Cherenkov (RV-C) [descoberta, em 1934, pelos físicos russos Pavel Alekseyvich Cherenkov (1904-1990; PNF, 1958) e Sergey Ivanovich Vavilov (1891-1951), em trabalhos independentes ${ }^{113}$, segundo a qual uma partícula carregada quando atravessa um meio transparente (de índice de refração $n$ ) com uma velocidade $\mathrm{V} / \mathrm{n}$ maior que a velocidade da luz no vácuo (c) $(\mathrm{V} / \mathrm{n}>\mathrm{c})$, ela perde uma fração de energia na forma de RV-C. Desse modo, por volta de 1980, Koshiba e colaboradores do ICRR construíram um tanque de 3.000 t de água, dotado de um detector Imagining Water Cherenkov (IWC), com fotomultiplicadores L (construídos e aperfeiçoados pela Hamamatsu Photonics Company) e o enterrou na mina de Mozumi, propriedade da Kamioka Mining and Smelting Company, na cidade de Hida (antiga Kamioka), em Gifu, no Japão, com 1.000 m de profundidade. Em vista disso, esse experimento foi denominado de KAMIOKANDE, onde NDE significa Nucleon Decay Experiment. Contudo, como nesse experimento foram detectados neutrinos atmosféricos, NDE passou a ser conhecido como Neutrino Detection Experiment. Ainda por volta de 1980, as Universidades de Irvine, Michigan e Brookhaven, sob a liderança de Reines, construíram o Nucleon Decay Detector (IMB/NDD), no terreno da Morton Salt Mine, localizada em Cleveland, no estado de Ohio, nos Estados Unidos. É interessante destacar que, em 1983114, Kajita e os físicos japoneses H. Kume, S. Sawaki, M. Ito, K. Arisaka, A. Nishimura e A. Suzuki descreveram um fotomultiplicador. Note-se que o espalhamento $\bar{v}_{e}-\mathrm{p}$ decorre da reação: $\bar{v}_{e}+\mathrm{p} \rightarrow \mathrm{e}^{+}+\mathrm{n}$, sendo o $\mathrm{e}^{+}$ detectado pela RV-C. Merece destaque o fato de que, o decaimento do próton (do tipo: $\mathrm{p} \rightarrow \mathrm{e}^{+}$ $+\pi^{0}$ ) foi examinado, também em $1983^{115}$ por Reines e seus 28 colaboradores, bem como, em $1985^{116}$, por Koshiba e seus 12 colaboradores (incluindo Kajita). Nessas duas experiências, foi

\footnotetext{
112 <en.wikipedia.org/wiki/Neutrino_Oscillation>.

113 Doklady Akademii Nauk SSSR 2, p. 451; 457, 1934.

114 Nuclear Instruments and Methods, v. 205, p. 443, 1983.

115 Physical Review Letters, v. 51, p. 27, 1983.

116 Journal of the Physical Society of Japan, v. 54, p. 3213, 1985.
} 
encontrado um limite superior para o tempo $\left(\tau_{\mathrm{p}}\right)$ de decaimento do próton: $\tau_{\mathrm{p}}>6,5 \times 10^{31}$ anos.

Com a observação da supernova (SN), ocorrida em 23 de fevereiro de 1987, na Grande Nebulosa de Magalhães, a hoje conhecida SN1987A, as duas colaborações KAMIOKANDE e IMB/NDD, passaram a detectar os neutrinos/antineutrinos atmosféricos oriundos dessa supernova observando o seu espalhamento em $\mathrm{p}$ e e da água. O espalhamento de $\bar{v}_{e}-\mathrm{p}$ foi publicado, em 1987, em trabalhos independentes, de Koshiba e 21 colaboradores (incluindo Kajita) ${ }^{117}$, e de Reines e 36 colaboradores ${ }^{118}$. Observe-se que resultado importante do experimento KAMIOKANDE foi encontrado, em 1988119, na qual Koshiba e seus 25 colaboradores (incluindo Kajita) anunciaram a descoberta da chamada Anomalia do Neutrino Atmosférico (ANA), ao observarem que a razão entre o número de neutrinos muônicos $\left[\mathrm{N}\left(v_{\mu}\right)\right]$ e o de neutrinos eletrônicos $\left[\mathrm{N}\left(v_{\mathrm{e}}\right)\right]$ era quatro (4) em vez de dois (2) conforme indicava o MPFPE.

Destaque-se que a observação de neutrinos eletrônicos oriundos de uma SN apresenta grande interesse de investigação, pois, durante sua longa viagem até nosso planeta podem ocorrer decaimentos (oscilações) permitindo, portanto, estimar um limite mínimo para a vida média dos mesmos.

Desse modo, com o objetivo de realizar novas observações dos neutrinos atmosféricos da SN1987A, em 1988 ${ }^{120}$, Koshiba e seus 23 colaboradores (incluindo Kajita), descreveram a construção de um detector constituído de um grande reservatório de água enterrado no terreno da Morton Salt Mine. Com tal detector, eles procuraram medir o decaimento de prótons da água, observando a colisão dos neutrinos atmosféricos da SN1987A com a água do detector. Também, em 1988 121 , Reines e 32 seus colaboradores mediram a vida média $\left(\tau_{\mathrm{p}}\right)$ do decaimento do próton, decorrente da passagem de neutrinos solares por toneladas de água, e encontraram que: $\tau_{\mathrm{p}}>10^{32}$ anos. Este mesmo valor e com esse mesmo tipo de experiência, foi encontrado, em $1989^{122}$, por Koshiba e seus 33 colaboradores (incluindo Kajita). É oportuno destacar que a proposta do decaimento do próton decorre da Teoria de Grande Unificação (TGU), proposta em $1972^{123}$ pelos físicos, o indiano Jogesh C. Pati (n.1937) e o paquistanês Salam e, em 1974124, pelos físicos norte-americanos Howard Mason Georgi (n.1947) e Glashow. Nessa TGU, $\tau_{\mathrm{p}}$ é da ordem de $10^{29} \pm 1,7$ anos, para altas energias $\left(\sim 10^{15} \mathrm{GeV}\right)$, correspondentes a dimensões da ordem de $10^{-29} \mathrm{~cm}$. Note-se que, segundo a Teoria do Big Bang (TBB), a idade do Universo, tem o seguinte valor: $\tau_{\mathrm{U}} \approx(13,73 \pm 0,15) \times 10^{6}$ anos, segundo observação realizada pelo Wilkinson

\footnotetext{
117 Physical Review Letters, v. 58, p. 1490, 1987.

118 Physical Review Letters, v. 58, p. 1494, 1987.

${ }^{119}$ Physics Letters, B205, p. 416, 1988.

${ }^{120}$ Physical Review, D38, p. 448, 1988.

${ }^{121}$ Physical Review Letters, v. 61, p. 2522, 1988.

122 Physics Letters, B220, p. 308, 1089.

123 Proceedings of the 16th Conference on High Energy Physics, 1972.

${ }^{124}$ Physical Review Letters, v. 32, p. 438, 1974.
} 
Microwave Anisotropy Probe (WMAP) e anunciada em março de 2006.

O espalhamento dos neutrinos atmosféricos por elétrons ( $\mathrm{e}^{-}$) da água foi observado, pela primeira vez, em 1989125, pela colaboração KAMIOKANDE/IMB (liderada por Koshiba e mais 32 colaboradores incluindo Kajita) e confirmado em $1990^{126}$, por essa mesma colaboração, também liderada por Koshiba e mais 36 colaboradores, ainda com a participação de Kajita.

Em dezembro de 1990, Koshiba preparou um artigo no qual apresentou um projeto para ampliar o KAMIOKANDE. Desta vez, o SUPER-KAMIOKANDE (S-K), como ficou conhecido, teria um tanque de água pura de 50.000 t e seria rodeado por cerca de 11.200 fotomultiplicadores, conforme descrição feita, em 1992, por Koshiba ${ }^{127}$ e pelo físico japonês Yoji Totsuka (1942-2008)128. Enquanto esse super detector não foi concluído (o que só aconteceu em 1996), o KAMIOKANDE juntou-se com alguns físicos do IMB e continuaram a busca pelos neutrinos atmosféricos e suas oscilações, usando a RV-C e o Standard Solar Model (SSM), formulado por J. N. Bahcall, a partir de 1962 (como assinalado antes). Assim, essa colaboração KAMIOKANDE/IMB (sob a liderança de Koshiba e a participação ativa de Kajita) divulgou esses resultados: em $1991^{129}$ (e mais 37 cientistas), ainda em $1991^{130}$ (e mais 33 cientistas); em $1992^{131}$ (e mais 39 cientistas); em 1994132 (e mais 52 cientistas); e em $1996^{133}$ (e mais 47 cientistas).

Com a entrada em funcionamento do S-K, em 1996 [iniciando a Astrofísica do Neutrino/Antineutrino, na qual foram usados métodos estatísticos constituindo o chamado gráfico do neutrino (neutrinograph)], novos resultados sobre a oscilação de neutrinos foram então encontrados por Koshiba e Kajita, em $1998^{134}$ (e mais 117 cientistas) e, ainda em 1998135, com a colaboração de mais 46 cientistas). Em 1999136, Koshiba, Kajita e Reines (e mais 117 cientistas), usaram o neutrinograph e concluíram que o fluxo de neutrinos atmosféricos observado era muito menor do que o previsto pelo SSM. Essa mesma discrepância foi encontrada pelo GNO,

\footnotetext{
125 Physical Review Letters, v. 63, p. 16, 1989.

126 Physical Review Letters, v. 65, p. 1297, 1990.

127 Physics Reports, v. 220, p. 229, 1992.

128 Reports on Progress in Physics, v. 55, p. 377, 1992.

${ }^{129}$ Physical Review, D44, p. 2241, 1991.

130 Physical Review Letters, v. 65, p. 1297, 1991.

131 Physical Review, D45, p. 2170, 1992.

132 Physics Letters, B335, p. 237, 1994.

133 Physical Review Letters, v. 77, p. 1683, 1996.

${ }^{134}$ Physical Review Letters, v. 81, p. 1562, 1998.

135 Physical Review Letters, v. 81, p. 2016, 1998.

136 Physical Review Letters, v. 82, p. 1810, 1999.
} 
em $2000^{137}$, em artigo assinado por 30 cientistas, dentre eles o nobelista Richter. Em 2002, novas observações sobre neutrinos solares foram apresentadas pelo SAGE ${ }^{138}$ (composto de 21 cientistas) e, também, por McDonald e mais 178 cientistas do GNO ${ }^{139}$. Note-se que a importância do trabalho de McDonald foi a de observar a contribuição das correntes neutras [que acontecem, por exemplo, em interações fracas (envolvendo a partícula $\mathrm{Z}^{0}$ ) do tipo: $\bar{v}_{e}+\mathrm{e}^{-} \rightarrow$ $\left.\bar{v}_{e}+\mathrm{e}^{-}\right]$como um argumento a mais que justifica a massa dos neutrinos/antineutrinos solares (João dos Anjos, e-mail, 04/12/2015).

É interessante salientar que, em 1999, foi criada a T2K Collaboration, constituída de 508 cientistas de 62 instituições de pesquisa em 12 países (Alemanha, Canadá, Coreia do Sul, Espanha, Estados Unidos da América, França, Inglaterra, Itália, Japão, Polônia, Rússia e Suíça), proposta pelos físicos japoneses Koichiro Nishikawa (n.1947) e Totsuka, tendo essa Colaboração (sob a direção de Kajita e mais 356 cientistas), em $2013^{140}$ observado, por intermédio do S$\mathrm{K}$, que neutrinos atmosféricos [eletrônicos $\left(v_{\mathrm{e}(1)}\right)$ e muônicos $\left(v_{\mu(2)}\right)$ ] se transformam em neutrinos tauônicos $\left(v_{\tau(3)}\right)$. Nesse experimento, foram encontrados os seguintes valores: $\operatorname{sen}^{2}\left(2 \theta_{32}\right)=$ $1, \delta_{\mathrm{CP}}=0 \mathrm{e}\left|\Delta \mathrm{m}^{2}{ }_{32}\right|=2,4 \times 10^{-3}\left(\mathrm{eV}^{2} / \mathrm{c}^{4}\right)$, valores esses que resultam em: $\operatorname{sen}^{2}\left(2 \theta_{13}\right)=$ $\left[0,088^{+0,49}{ }_{-0,039}\right.$ (estatístico + sistemático) $]$, com 3,1 $\sigma$ de confiabilidade. Registre-se que, em 18 de maio de 2015, por ocasião de um seminário no KEK High Energy Accelerator Research Organization (instalado em 1997, em Tsukuba, na Prefeitura de Ibaraki, no Japão), o T2K Collaboration anunciou que havia observado a oscilação de antineutrinos muônicos-tauônicos com os parâmetros oscilatórios: $\operatorname{sen}^{2}\left(2 \theta_{\overline{2} \overline{3}}\right)$ e $\Delta m^{2} \overline{3} \overline{2}$ com as respectivas confiabilidades: $68 \%$ e $90 \% 141$.

Por sua vez, com o objetivo de detectar antineutrinos eletrônicos $\left(\bar{v}_{e}\right)$, foi construído um novo detector - o Kamioka Liquid Scintillator Antineutrino Detector (KamLAND) - localizado também na Kamioka Mining. Ele foi preparado para detectar antineutrinos nucleares ( $\bar{v}_{e}$ ) (produzidos por reatores nucleares), bem como detectar neutrinos/antineutrinos atmosféricos/solares e neutrinos/antineutrinos geológicos - estes provenientes do decaimento radioativo do tório (Th) e do urânio (U) da crosta e do manto terrestres -, assim como a sua oscilação. Esse detector é constituído de um balão esférico de aço inoxidável, de $18 \mathrm{~m}$ de diâmetro, e contendo em seu interior 1.879 fotomultiplicadores. No interior dessa esfera existe um balão de nylon, de 13 m de diâmetro, cheio de um líquido cintilador composto de 1.000 t de óleo mineral, benzeno e fluorescentes químicos. Por sua vez, o balão de aço inoxidável é envolvido por um Detector Vavilov-Cherenkov, cuja finalidade é a de filtrar os neutrinos muônicos $\left(v_{\mu}\right)$ e a de

\footnotetext{
137 Physics Letters, B490, p. 15, 2000.

138 Journal of Experimental and Theoretical Physics, v. 95, p. 181, 2002.

${ }^{139}$ Physical Review Letters, v. 89, 011301, 2002.

${ }^{140}$ Physical Review, D88, n. 072010, 2013.

141 Physical Review, D88, n. 072010, 2013.
} 
proporcionar blindagem contra a radioatividade das rochas.

A Colaboração KamLAND (en.wikipedia.org/wiki/KamLand) começou a funcionar em janeiro de 2002 e, em 17 de janeiro de 2003142, 97 de seus cientistas anunciaram que haviam detectado um fluxo de $\bar{v}_{e}$, com energia $>3,4 \mathrm{MeV}$, oriundos de reatores nucleares que se localizavam em uma faixa de $180 \mathrm{~km}$. Já em 2004143, o KamLAND (com 90 cientistas) encontrou um limite superior do fluxo de antineutrinos atmosféricos, dado por: $3,7 \times 10^{2} /\left(\mathrm{cm}^{2}\right.$.s). Já a oscilação de neutrinos foi observada pelo KamLAND em duas oportunidades: 1) em $2005^{144}$ (93 cientistas); 2) em $2008^{145}$ (81 cientistas). Destaque-se que, em 2005 ${ }^{146}$, 97 pesquisadores daquela Colaboração publicaram o resultado de sua investigação experimental sobre os antineutrinos geológicos ${ }^{147}$.

Vejamos agora os experimentos com neutrinos/antineutrinos produzidos por aceleradores nucleares. Como vimos acima, o FERMILAB havia construído, em 1983, um acelerador de prótons (Tevatron) para estudar a colisão dessas partículas com alvos de grafite (para produzir píons e káons) e observar seus decaimentos envolvendo neutrinos $\left(v_{\mu}, v_{\tau}\right)$ e, então, produzir seus respectivos feixes [NuMI $\left(v_{\mu}\right)$, NuTAU $\left(v_{\tau}\right)$ ], constituindo-se nos denominados Main Injector Neutrino. Além do mais e, também, como destacamos anteriormente, em 1986, foi elaborada a Teoria das Oscilações de Neutrinos (matriz: PMNS). Desse modo, para medir a ON, o FERMILAB organizou o experimento denominado de Main Injector Neutrino Oscillation Search (MINOS), um sistema composto de dois detectores para receber aqueles feixes. Um deles [far detector (FD)], com massa de 5.400 t, localizado na Soudan Mine, no norte de Minnesota, na profundidade de 716 m, entrou em operação, no verão de 2003, para estudar os raios cósmicos e os neutrinos atmosféricos (estelares). O outro [near detector (ND)], de $980 \mathrm{t}$, distante de algumas centenas de metros do alvo de grafite, enterrado na profundidade de quase 100 $\mathrm{m}$, começando a funcionar em dezembro de 2004. Note-se que a distância entre esses dois detectores é de $734 \mathrm{~km}$.

A MINOS Collaboration trata da oscilação $\left[v_{\mu(2)} \rightarrow v_{\tau(3)}\right]$ e mede a diferença dos quadrados da massa $\left(\Delta \mathrm{m}^{2}{ }_{32}\right)$ e os ângulos de mistura $\left(\theta_{32}\right)$. Assim, seus resultados foram apresentados em: $2006^{148}$ [275 físicos, sendo 02 brasileiros: Carlos Ourivio Escobar (n.1948), do Instituto de Física da Universidade Estadual de Campinas (IF/UNICAMP) e Philippe Gouffon (n.1954), do Instituto de Física da Universidade de São Paulo (IF/USP)] e 2008 (Physical Review D77, no. 072002) (199 cientistas, incluindo Escobar e Gouffon), com os mesmos valores,

\footnotetext{
142 Physical Review Letters, v. 90, 021802, 2003.

143 Physical Review Letters, v. 92, n. 071301, 2004.

144 Physical Review Letters, v. 94, n. 081801, 2005.

145 Physical Review Letters, v. 100, n. 221803, 2008.

146 Nature, v. 436, p. 499, 2005.

147 <www.searadaciencia.ufc.br/bassalo/PNF2002>.

148 Physical Review Letters, v. 97, n. 191801, 2006.
} 
dados por: $\Delta \mathrm{m}_{32}^{2}=2,74^{+0,44}{ }_{-0,26} \times 10^{-3}\left(\mathrm{eV}^{2} / \mathrm{c}^{4}\right)$ e $\operatorname{sen}^{2}\left(2 \theta_{32}\right)>0,87$ [com $68 \%$ de confidence limit ("limite de confiança") (CL)]. Ainda em $2008^{149}$ (171 cientistas, também com Escobar e Gouffon), a MINOS voltou a apresentar novos resultados: $\Delta \mathrm{m}_{32}^{2}=2,43^{+0,13}-0,13 \times 10^{-3}\left(\mathrm{eV}^{2} / \mathrm{c}^{4}\right)$ e $\operatorname{sen}^{2}\left(2 \theta_{32}\right)>0,90$ (com 90\% de CL). Em $2011^{150}$ [128 cientistas, com Escobar e Gouffon, e mais dois brasileiros: João de Abreu Barbosa Coelho, do IF/UNICAMP e Ricardo Avelino Gomes (n.1976), do Instituto de Física da Universidade Federal de Goiás (IF/UFG)], os novos valores foram: $\Delta \mathrm{m}^{2}{ }_{32}=2,32^{+0,12}{ }_{-0,08} \times 10^{-3}\left(\mathrm{eV}^{2} / \mathrm{c}^{4}\right)$ e $\operatorname{sen}^{2}\left(2 \theta_{32}\right)>0,90$ (com 90\% de CL). Ainda em 2011, a MINOS divulgou novos resultados, desta vez envolvendo o desaparecimento de antineutrinos muônicos $\left(\bar{v}_{\mu}\right)$ em dois artigos. O primeiro foi publicado no Physical Review

Letters, 107, n. 021801 (128 cientistas, e mais Coelho, Escobar, Gomes e Gouffon), cujos resultados foram os seguintes valores: $\Delta m_{\mu \bar{\mu}}^{2}=\left[2,43^{+0,13}{ }_{-0,13}\right.$ (estatístico) $\pm 0,06$ (sistemático) $] \times$ $10^{-3}\left(\mathrm{eV}^{2} / \mathrm{c}^{4}\right)$, além de $\operatorname{sen}^{2}\left(2 \theta_{\mu \bar{\mu}}\right)=\left[0,86^{+0,11}{ }_{-0,12}\right.$ (estatístico) $\pm 0,01$ (sistemático) $]$. Este experimento, que também mediu a oscilação $\left(v_{\mu} \leftrightarrow \bar{v}_{\mu}\right)$, é consistente no nível de $2 \%$ ao assumir parâmetros de oscilação idênticos. O segundo, agora apresentado no Physical Review, D84, no. 071103 (124 cientistas, incluindo Coelho, Escobar, Gomes e Gouffon), os resultados são dados por: $\Delta m_{\mu \bar{\mu}}^{2}=2,32 \times 10^{-3}\left(\mathrm{eV}^{2} / \mathrm{c}^{4}\right)$ e $\operatorname{sen}^{2}\left(2 \theta_{\mu \bar{\mu}}\right)=1$.

Como os dois últimos experimentos de 2011 indicavam uma possível violação da $\mathrm{Si}$ metria (Teorema) CPT [inversão de carga $(\mathrm{C})$ : nenhuma experiência física se altera se uma partícula $(\mathrm{p})$ for trocada por sua antipartícula $(\bar{p})$; inversão espacial ou paridade $(\mathrm{P})$ : nenhuma experiência física será capaz de determinar, de maneira unívoca, a direita ou a esquerda (troca de $\mathrm{r}$ por - r); e inversão temporal (T): se um certo movimento de sistema físico é possível, o seu reverso temporal também o será (troca de t por - t)], e que é um pilar básico do MPPE, a MINOS voltou a realizar nova experiência, em 2012 ${ }^{151}$ [112 cientistas, ainda com Coelho, Escobar, Gomes e Gouffon), não confirmou o observado no artigo de 2011 ${ }^{152}$, ou seja, uma possível violação da CPT. Desse modo, essa experiência de 2012 mostrou que a Simetria CPT continua válida no MPFPE, pois os parâmetros para neutrinos e antineutrinos se mostraram consistentes. Nesta altura, é interessante registrar que, em $1964^{153}$, os físicos, os norte-americanos James Henry Christenson (1935-2015), James Watson Cronin (n.1931; PNF, 1980), Val Lodgsdon Fitch (1923-2015; PNF, 1980) e o francês René Turlay (1932-2002) observaram a violação da simetria $C P$ do sistema $K_{0}-\bar{K}_{0}$ (que são partículas neutras e massivas), porém, havia a preservação da CPT.

No caso dos experimentos de 2011 tratados acima, existem dois pilares básicos do

\footnotetext{
149 Physical Review Letters, v. 101, n. 131802, 2008.

150 Physical Review Letters, v. 106, n. 181801, 2011.

151 Physical Review Letters, v. 108, n. 191801, 2012.

152 Physical Review Letters, v. 106, n. 181801, 2011.

153 Physical Review Letters, v. 13, p. 138, 1964.
} 
MPPE que estão afetados: os neutrinos parecem possuir massa e sua oscilação viola a CPT. Portanto, se isso se confirmar no futuro, há a necessidade de se revisar o MPPE.

Ainda se tratando da MINOS Collaboration, merece destaque o fato de que ela esteve no centro das polêmicas experiências envolvendo a massa e a velocidade dos neutrinos, como, por exemplo, a realizada em 2007154 (203 cientistas, com Escobar e Gouffon). Neste experimento foi usado um feixe de neutrinos muônicos (NuMI) com a energia da ordem de $3 \mathrm{GeV}$ (com uma determinada velocidade $\mathrm{v}$ para o $v_{\mu}$ ), tendo o far detector detectado 473 eventos, para os quais foi encontrado: $\mathrm{v}=1,000051 \mathrm{c}$ (com 68\% de CL). Por sua vez, as medidas de correlação entre as massas dos $v_{\mu}$ e correspondentes a 258 eventos, mostrou que o limite de massa imposto para esses neutrinos foi de: $\mathrm{m}<50 \mathrm{MeV} / \mathrm{c}^{2}$ (com 99\% de CL). Para esses mesmos 258 eventos, foi encontrado que: $0,999976 \mathrm{c}<\mathrm{v}<1,000126 \mathrm{c}$. Registre-se que em 15 de setembro de 2015 155 , ainda a MINOS (123 cientistas, com Coelho, Escobar, Gomes e Gouffon) obteve o seguinte resultado: $\mathrm{v} / \mathrm{c}-1=(1,0 \pm 1,1) \times 10^{-6}$, consistente com $\mathrm{v}<\mathrm{c}^{156}$.

Segundo vimos acima, as experiências que examinaram a oscilação de neutrinos envolvem a medida de ângulos de mistura $\left(\theta_{\mathrm{ij}}\right)$. Assim, para os neutrinos solares, são medidos: $\theta_{12}$ [mistura de neutrinos eletrônicos $\left(v_{\mathrm{e}}\right)$ e de muônicos $\left(v_{\mu}\right)$ ]; para os neutrinos atmosféricos (estelares), tem-se: $\theta_{23}$ [mistura de neutrinos muônicos $\left(v_{\mu}\right)$ e de tauônicos $\left(v_{\tau}\right)$ ]; e para os neutrinos nucleares, medem-se: $\theta_{13}$ [mistura de neutrinos eletrônicos $\left(v_{\mathrm{e}}\right)$ e de tauônicos $\left(v_{\tau}\right)$ ]. Para a medida de $\theta_{13}$, foram realizadas (em 2012) três experiências: Double Chooz Collaboration (França), Daya Bay Collaboration (China) e RENO Collaboration (Coreia). Vejamos os resultados de cada uma delas ${ }^{157}$.

A Double Chooz Collaboration (DCC) foi um experimento localizado no município de Chooz, região de Ardennes (França perto da Bélgica) e destinado a medir o desaparecimento do antineutrino eletrônico nuclear $\left(\bar{v}_{e}\right)$ cujos fluxos eram produzidos por dois reatores [4,25 GWe (1 GWe = $10^{9}$ watts) ] da Chooz Nuclear Power Plant, oscilação essa medida por um detector (far detector) distante de $1.050 \mathrm{~m}$ dos reatores e localizado em cavernas de uma colina. Seu objetivo era medir o limite do parâmetro $\theta_{13}$ (cuja expressão analítica foi acima registrada), sendo este consistente com a oscilação $\left(\bar{v}_{e(1)} \leftrightarrow \bar{v}_{\tau(3)}\right)$. Assim, esse limite foi medido ${ }^{158}$ pela DCC, da qual participaram 184 cientistas (físicos e engenheiros), de 36 instituições de pesquisa mundiais: 12 americanas; 07 japonesas; 05 francesas; 05 alemães; 02 russas; 01 espanhola, 01 inglesa e 03 brasileiras [João Carlos Costa dos Anjos (n.1944) (a quem agradeço nesta oportunidade a leitura crítica deste artigo), Ademarlaudo França Barbosa (1963-2012), Rafael Gonçalves Gama (n.1984), Herman Pessoa Lima Júnior (n.1973) e Iuri Muniz Pepe

\footnotetext{
154 Physical Review, D76, n. 072005, 2007.

155 Physical Review, D92, n. 052005), 2015.

156 Ricardo Gomes, e-mail, 12/02/2016; <en.wikipedia.org/MINOS>.

157 <en.wkipedia.org/Double_Chooz/Daya_Bay/RENO>.
}

158 Physical Review Letters, v. 108, n. 131801, 28/03/2012. 
[agora, na Universidade Federal da Bahia (UFBA)], do Centro Brasileiro de Pesquisas Físicas (CBPF); Pietro Chimenti, da Universidade Federal do ABC (UFABC); Luis Fernando Gomez Gonzalez e Ernesto Kemp, do Instituto de Física da Universidade Estadual de Campinas (IF/UNICAMP)]. O resultado obtido nesse experimento foi o seguinte: $\operatorname{sen}^{2}\left(2 \theta_{13}\right)=$ $[0,086 \pm 0,041$ (estatístico) $\pm 0,030$ (sistemático)], o que corresponde a $\sim 3 \sigma(\sigma=$ desvio padrão) para $\theta_{13} \neq 0$. É oportuno observar que, em setembro de 2014, foi instalado mais um detector (near detector) na DCC, distante $400 \mathrm{~m}$ dos reatores.

A Daya Bay Collaboration (DBC) corresponde a um experimento realizado na península Dapeng, na costa oeste da Baia Daya, no sul da China, projetado também para medir o desaparecimento de $\bar{v}_{e}$ sendo seus fluxos oriundos de seis (6) reatores nucleares $(2,9 \mathrm{GWe})$ produzidos pelo Daya Bay Reactor Neutrino Experiment (operado por 271 cientistas de 38 institutos de pesquisa: 20 chineses; 16 norte-americanos; 01 russo e 01 techcolosváquio) e examinados por 6 detectores enterrados na profundidade de $1.648 \mathrm{~m}$, nos quais foi medido o $\theta_{13}$. Seu resultado ${ }^{159}$, apresentou o seguinte valor: $\operatorname{sen}^{2}\left(2 \theta_{13}\right)=[0,092 \pm 0,016$ (estatístico) $\pm 0,005$ (sistemático)] e equivalente a 5,2 $\sigma$ para a existência do $\theta_{13}$.

Por sua vez, a RENO (Reactor Experiment for Neutrino Oscillations) Collaboration estabeleceu-se no então Yeonggwang Nuclear Power Plant (YNPP) [cujo nome foi mudado para Hanbit Nuclear Power Plant (HNPP), em 2013], na província Jeollanam, na Coreia do Sul e com o mesmo propósito das duas anteriores, ou seja: medir o $\theta_{13}$. Desse modo, um fluxo de antineutrinos eletrônicos $\left(\bar{v}_{e}\right)$ produzido por seis $(6)$ reatores nucleares $(2,8 \mathrm{GWe})$ foi registrado em dois detectores idênticos e distantes, de $294 \mathrm{~m}$ e $1.383 \mathrm{~m}$, do complexo YNPP (no qual participaram 34 cientistas de 12 instituições de pesquisa sul-coreanas). Seu resultado ${ }^{160}$ foi: $\operatorname{sen}^{2}\left(2 \theta_{13}\right)=[0,113 \pm 0,013$ (estatístico) $\pm 0,019$ (sistemático) $]$ consistente com a oscilação $\left(\bar{v}_{e(1)} \leftrightarrow \bar{v}_{\tau(3)}\right)$, com $4,9 \sigma$ de significância para a existência do $\theta_{13}$.

Para mais informações sobre o tema do PNF/2015, ver: Gustavo do Amaral Valdiviesso e Marcelo M. Guzzo, Compreendendo a Oscilação de Neutrinos, Revista Brasileira de Ensino de Física 27, p. 495 (2005); Ricardo Avelino Gomes, Olhando o Céu do Fundo de um Poço, Revista UFG 8, p. 101 (2010); e Ricardo Avelino Gomes, Neutrinos em Tudo que Eu Vejo, Revista UFG 12, p. 245 (2012).

Antes de concluir este artigo, é interessante registrar mais dois aspectos relacionados com o tema do PNF/2015. O primeiro refere-se a dois trabalhos realizados em 2015 e que tratam da massa dos neutrinos/antineutrinos e de um possível decaimento deles. Com efeito, em janeiro de 2015 ${ }^{161}$, o Prof. Ricardo Gomes e seus colaboradores, os físicos brasileiros Abner Leonel Gadelha Gomes (n.1990) e Orlando Luís Goulart Peres (n.1969), realizaram um trabalho no qual examinaram os resultados dos experimentos do MINOS e do T2K sobre a relação entre

\footnotetext{
159 Physical Review Letters, v. 108, n. 171803, 23/04/2012.

${ }^{160}$ Physical Review Letters, v. 108, n. 191802, 11/05/2012.

161 Physics Letters, B740, p. 345, 2015.
} 
o tempo $\left(\mathrm{T}_{3}\right)$ de decaimento do $v_{3}$ (representando os neutrinos massivos 1,2 e 3 ) e sua massa $\left(\mathrm{m}_{3}\right)$, considerando correntes neutras (envolvendo $\mathrm{Z}^{0}$ ) e correntes carregadas (evolvendo $\mathrm{W}^{ \pm}$), eles encontraram que (considerando $\mathrm{c}=1$ ): $\mathrm{T}_{3} / \mathrm{m}_{3}>2,8 \times 10^{-12} \mathrm{~s} / \mathrm{eV}$, com $90 \%$ de CL, porém com baixa significância $(\sigma)$. Por sua vez, em setembro de $2015^{162}$ (97 cientistas), a Borexino Collaboration, além de confirmar a existência do neutrino massivo, estimou o melhor limite da vida média do elétron: $\tau_{\mathrm{e}}>6,6 \times 10^{28}$ anos, com $90 \%$ de CL [lembrar que: $\tau_{\mathrm{p}} \approx 10^{29} \pm 1,7$ anos e $\tau_{\mathrm{U}} \approx(13,73 \pm 0,15) \times 10^{6}$ anos], caso o elétron apresentasse o seguinte decaimento: $\mathrm{e}^{-} \rightarrow v_{\mathrm{e}}+\gamma$. [que, no entanto, ainda não foi observado (Ricardo Gomes, e-mail, 25/01/2016)].

$\mathrm{O}$ segundo trata da continuação dos trabalhos sobre os "misteriosos neutrinos" e suas oscilações como, por exemplo, por parte do FERMILAB e, também, do Brasil. No FERMILAB, a linha de feixe NuMI, que fornecia o feixe de neutrinos para o experimento MINOS, foi modificada para atender ao experimento NuMI Off-Axis ve Appearance (NOvA), a partir de 2013, que tem as seguintes metas a serem atingidas até 2020: 1) Determinar a hierarquia de massa dos neutrinos; 2) Determinar o octante do $\theta_{23}$; 3) Investigar a violação do CP dos léptons; e 4) Procurar por possível neutrino estéril. Essa Colaboração conta com a presença da Universidade Federal de Goiás (UFG), sob a coordenação Prof. Ricardo Avelino Gomes e mais dois físicos, o brasileiro Stefano Castro Tognini (n.1987) e a indiana Tapasi Ghosh (n.1981). (Ricardo Gomes, e-mails, 15/01/2016, 12/02/2016 e 15/02/2016).

A participação de físicos brasileiros na Double Chooz Collaboration (DCC) sobre a oscilação de antineutrinos eletrônicos nucleares e vista anteriormente, ensejou que o Brasil desenvolvesse também um projeto próprio para tratar do mesmo tema e usando o reator de 4 GWe da Usina Angra dos Reis II (UAR/II), que produz $10^{22}$ antineutrinos eletrônicos e um fluxo de $10^{12}$ antineutrinos/s. Assim, os professores Ernesto Kemp (UNICAMP) e João dos Anjos (CBPF) (ex-participantes da DCC), começaram, em 2005, a preparar o Projeto Brasileiro de Neutrinos, constituído de um detector pequeno e de outro muito maior de 50 t de água ultrafiltrada, a ser enterrado no Morro do Frade, distante de 1,5 km da UAR/II. Contudo, devido ao elevado custo (US\$50 milhões) do detector maior, esses professores decidiram, inicialmente, construir o detector menor e, para isso, envolveram mais 25 físicos das seguintes universidades: Universidade Estadual de Campinas (UNICAMP) (Gonzalez, ex-participante da DCC; Ohana Benevides Rodrigues; e L. M. Santos); Universidade Estadual de Feira de Santana (UEFS/BA) (Germano Pinto Guedes); Universidade Federal do ABC (UFABC/SP) (Chimenti, ex-participante da DCC); Universidade Federal de Alfenas (UNIFAL/MG) (L. P. Andrade; A. L. M. Reis; S. M. V. Santos; e Gustavo do Amaral Valdiviesso); Universidade Federal da Bahia (UFBA) [Pepe, ex- participante da DCC; P. C. M. A. Farias; D. B. S. Ribeiro; e Eduardo Furtado de Simas Filho (n.1978)]; Universidade Federal do Oeste da Bahia (UFOB) (M. J. N. Souza); Universidade Federal de Juiz de Fora (UFJF/MG) (T. A. Alvarenga; A. S. Cerqueira; J. A. Costa; T. I. Dornelas; e R. A. Nobrega) e, também, do CBPF [Lima, ex-participante da

162 Physical Review, v. 115, n. 231802, 2015. 
DCC; Thamys Abrahão (n.1987); Gabriel Azzi; Fernando Ferreira de França (n.1981); Rogério Machado da Silva (n.1971); e Stefan Wagner (n.1983)].

$\mathrm{O}$ pequeno detector de antineutrinos eletrônicos idealizado pelo grupo de físicos brasileiros referido anteriormente (colocado a $30 \mathrm{~m}$ da UAR/II), é do tipo RV-C ("Radiação de Vavilov-Cherenkov"), tem o volume de $1,42 \mathrm{~m}^{3}$, envolvido de $50 \mathrm{~cm}$ de água ultrafiltrada, colocado em um contêiner de $12 \mathrm{~m}$, cercado por 32 fotomultiplicadores [dispositivos CCD ("Charge-Coupled Device"), amplamente utilizados em Astronomia, mas que apenas recentemente têm sido explorados para detectar neutrinos/antineutrinos e matéria escura (esta constitui cerca de $70 \%$ da matéria do Universo)].

Note-se que esse pequeno detector também é usado para monitorar as atividades de reatores nucleares sem precisar ter acesso a ele. Desse modo, surgiu o Experimento Neutrinos Angra (ENA), cujo primeiro resultado foi o artigo Using Neutrinos to Monitor Nuclear Reactors: the Angra Neutrino Experiment, Simulation and Detector Status e apresentado no X Latin American Symposium of High Energy Physics, que aconteceu em Medellín, Colômbia, no período 24-28 de novembro de 2014, e publicado no Nuclear and Particle Physics Proceedings 267-269, p. 108-115, October-December 2015. (Inovações Tecnológicas, 30/01/2016).

Com a experiência adquirida no ENA, o CBPF criou o Coherent Neutrino-Nucleus Interaction Experiment (CONNIE), sob a coordenação do Prof. João dos Anjos e com a colaboração dos físicos brasileiros Martin Makler (n.1974), Hélio da Motta Filho (n.1955) e Herman Lima, e mais a participação de Carla Brenda Bonifazzi (n.1975), da Universidade Federal do Rio de Janeiro (UFRJ). Um dos objetivos do CONNIE é a realização da interação coerente de neutrinos, prevista pelo MPFPE e ainda não conseguida em nenhum laboratório do mundo. Destaque-se que, entre os dias 09 e 12 de junho de 2015, no CBPF, o CONNIE realizou o I CONNIE Collaboration Meeting ("Primeiro Encontro da Colaboração CONNIE"), com a participação de pesquisadores de instituições da Argentina, Brasil, Estados Unidos, México e Paraguai, no qual foi discutido o que já foi realizado pelo CONNIE e seu planejamento para as próximas etapas ${ }^{163}$.

Na conclusão deste artigo sobre o PNF/2015, que mostrou as evidências observacionais sobre o neutrino/antineutrino (atmosféricos e solares) e suas oscilações, destaquemos que elas apresentam dois resultados surpreendentes contra o MPFPE: 1) neutrinos massivos; 2) não conservação do CPT. Além disso, existe uma hipotética - não conservação da carga elétrica e que, também, não é prevista pelo MPFPE. Portanto, tais resultados indicam que neste Século 21 darão muito trabalho para os cientistas-filósofos em busca de uma NOVA FÍSICA. Mas, perguntamos: existe uma NOVA MATEMÁTICA para apoiá-la como aconteceu até hoje?

163 <en.wikipedia.org/wiki/CONNIE>; João dos Anjos, e-mail, 10/12/2015. 